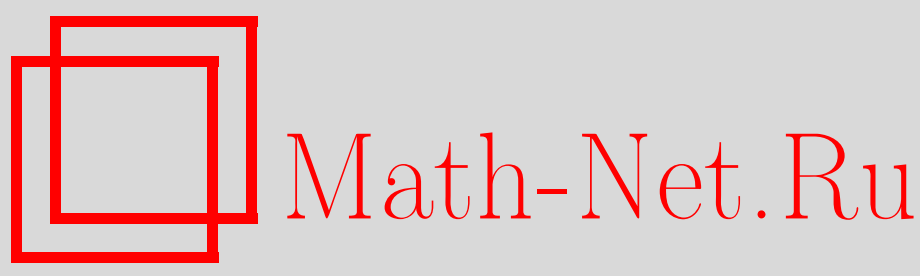

Л. И. Данилов, О почти периодических мерозначных функциях, Матем. сб., 2000, том 191, номер 12, 27-50

DOI: https://doi.org/10.4213/sm527

Использование Общероссийского математического портала Math-Net.Ru подразумевает, что вы прочитали и согласны с пользовательским соглашением

http: //www. mathnet.ru/rus/agreement

Параметры загрузки:

IP : 54.162 .127 .20

26 апреля 2023 г., $12: 12: 43$ 


\section{О почти периодических мерозначных функциях}

Рассматриваются слабо почти периодические мерозначные функции $\mathbb{R} \ni t \rightarrow$ $\mu[\cdot ; t]$ со значениями в пространстве $\mathscr{M}(U)$ борелевских знакопеременных мер на полном сепарабельном метрическом пространстве $U$. На линейном пространстве $\mathscr{M}(U)$ вводится норма $\|\cdot\|_{w}$, которая на множестве вероятностных борелевских мер определяет метрику, эквивалентную метрике Леви-Прохорова. Устанавливается связь между почти периодичностью мерозначной функции $t \rightarrow \mu[\cdot ; t] \in$ $\left(\mathscr{M}(U),\|\cdot\|_{w}\right)$ и ее слабой почти периодичностью (как по Бору, так и по Степанову).

Библиографиия: 20 названий.

Пусть $(U, \rho)$ - полное сепарабельное метрическое пространство, $\mathscr{M}(U)$ - линейное пространство борелевских знакопеременных мер (зарядов), определенных на $\sigma$-алгебре $\mathscr{B}(U)$ борелевских множеств $\mathscr{O} \subset U$, наделенное нормой

$$
\|\mu\|_{w}=\sup _{F \in \mathscr{L}(U)}\left|\int_{U} F(x) \mu[d x]\right|, \quad \mu[\cdot] \in \mathscr{M}(U),
$$

где $\mathscr{L}(U)$ - множество функций $U \ni x \rightarrow F(x) \in \mathbb{R}$ таких, что $0 \leqslant F(x) \leqslant 1$ и $|F(x)-F(y)| \leqslant \rho(x, y)$ для всех $x, y \in U$. Обозначим через $\mathscr{M}_{0}(U)$ множество вероятностных борелевских мер из $\mathscr{M}(U)$. На множестве $\mathscr{M}_{0}(U)$ норма $\|\cdot\|_{w}$ определяет метрику, эквивалентную метрике Леви-Прохорова $\rho_{0}[1 ;$ с. 377].

Пусть $C A P(\mathbb{R}, \mathscr{M}(U))$ и $S_{p}(\mathbb{R}, \mathscr{M}(U))$ - множества почти периодических (п.п.) по Бору и п.п. по Степанову степени $p \geqslant 1$ мерозначных функций $\mathbb{R} \ni t \rightarrow \mu[\cdot ; t]$ со значениями в линейном нормированном пространстве $\left(\mathscr{M}(U),\|\cdot\|_{w}\right)$. Мерозначная функция $\mathbb{R} \ni t \rightarrow \mu[\cdot ; t] \in \mathscr{M}(U)$ называется слабо почти периодической по Бору (слабо n.n. по Степанову степени $p \geqslant 1$ ), если для любой непрерывной ограниченной функции $F \in C_{b}(U)=C_{b}(U, \mathbb{R})$ функция $t \rightarrow \int_{U} F(x) \mu[d x ; t]$ п.п. по Бору (п.п. по Степанову степени $p$ ). Соответствующие множества слабо п.п. функций обозначим через $C A P^{w}(\mathbb{R}, \mathscr{M}(U))$ и $S_{p}^{w}(\mathbb{R}, \mathscr{M}(U))$.

Мерозначные функции используются в задачах теории управления [2]-[4]. Слабо п.п. по Степанову мерозначные функции при исследовании оптимального п.п. управления рассматривались в [5], [6]. В [7] доказано, что многозначные отображения $\mathbb{R} \ni t \rightarrow \operatorname{supp} \mu[\cdot ; t]$, где $\mu[\cdot ; \cdot] \in S_{1}\left(\mathbb{R}, \mathscr{M}_{0}(U)\right)$, и только они представимы в виде $\overline{\bigcup_{j \in \mathbb{N}} f_{j}(t)}, t \in \mathbb{R}$, где $f_{j}(\cdot)$ - п.п. по Степанову функции (supp $\mu$ - носитель меры $\mu \in \mathscr{M}_{0}(U), \bar{A}$ - замыкание множества $\left.A \subset U\right)$. В ряде задач возникает необходимость использовать также функции $\mu[\cdot ; t]$ со знакопеременной мерой (в частности, при изучении систем интегро-дифференциальных уравнений [8; приложение А], систем дифференциальных уравнений с распределенным запаздыванием [9], а также в задачах управления системами с распределенными параметрами [10]).

(C) Л.И. ДАнилов 2000 
В настояшей работе рассматриваются слабо почти периодические мерозначные функции $\mathbb{R} \ni t \rightarrow \mu[\cdot ; t] \in \mathscr{M}(U)$. Устанавливается связь между слабой почти периодичностью функции $t \rightarrow \mu[\cdot ; t]$ и почти периодичностью этой функции со значениями в линейном нормированном пространстве $\left(\mathscr{M}(U),\|\cdot\|_{w}\right)$.

В $\S 1$ приведены определения и сформулирован ряд утверждений, которые существенно используются в дальнейшем. Относительно утверждений о п.п. функциях см. [11], [12], о борелевских мерах - [13]. Основные результаты содержатся во втором и третьем параграфах. Пусть $\mathscr{M}^{+}(U)$ - множество неотрицательных мер из $\mathscr{M}(U),|\mu| \in \mathscr{M}^{+}(U)$ - полная вариация меры $\mu \in \mathscr{M}(U)$. В $\S 2$ доказано, что мерозначная функция $\mathbb{R} \ni t \rightarrow \mu[\cdot ; t] \in \mathscr{M}(U)$ слабо п.п. по Бору тогда и только тогда, когда $\mu[\cdot ; \cdot] \in C A P(\mathbb{R}, \mathscr{M}(U))$ и найдется компакт $K \subset\left(\mathscr{M}(U),\|\cdot\|_{w}\right)$ такой, что $|\mu|[\cdot ; t] \in K$ при всех $t \in \mathbb{R}$. Доказательство основано на следующем утверждении (которое вытекает из теоремы 2).

ПреДЛОЖЕНИЕ 1 . Пусть $(\mathscr{H},\|\cdot\|)$ - банахово пространство $u f_{j}: \mathbb{R} \rightarrow \mathscr{H}$, $j \in \mathbb{N},-$ последовательность функиий таких, что при всех $t \in \mathbb{R}$ ряд $\sum_{j \in \mathbb{N}} f_{j}(t)$ безусловно сходится. Предположим, что для всех (непустых) многеств $J \subset \mathbb{N}$ функиии $t \rightarrow \sum_{j \in J} f_{j}(t)$ n.n. по Бору. Тогда

$$
\sup _{t \in \mathbb{R}}\left\|\sum_{j \geqslant l} f_{j}(t)\right\| \rightarrow 0 \quad \text { npu } \quad l \rightarrow+\infty .
$$

Аналогичное утверждение (являющееся следствием теоремы 4) справедливо также для п.п. по Степанову функций $f_{j} \in S_{p}(\mathbb{R}, \mathscr{H}), j \in \mathbb{N}, p \geqslant 1$.

ПреДЛОЖЕНИЕ 2. Пусть $(\mathscr{H},\|\cdot\|)$ - банахово пространство $u f_{j}: \mathbb{R} \rightarrow \mathscr{H}$, $j \in \mathbb{N},-$ последовательность функиий таких, что для любого (непустого) множества $J \subset \mathbb{N}$ ряд $\sum_{j \in J} f_{j}(t)$ при почти всех $t \in \mathbb{R}$ (за исключением множества нулевой мерьи, зависящего от $J$ ) сходится к некоторой функиии $f(J ; t)$ из $S_{p}(\mathbb{R}, \mathscr{H})$. Тогда

$$
\|f(\{j \in \mathbb{N}: j \geqslant l\} ; \cdot)\|_{S_{p}(\mathbb{R}, \mathscr{H})} \rightarrow 0 \quad \text { npu } \quad l \rightarrow+\infty
$$

В [14] была доказана эквивалентность понятий почти периодичности и слабой почти периодичности (как по Бору, так и по Степанову) для мерозначных функций $\mathbb{R} \ni t \rightarrow \mu[\cdot ; t] \in\left(\mathscr{M}_{0}(U), \rho_{0}\right)$. При доказательстве использовались частный случай предложения 1 для пространства $\mathscr{H}=\mathbb{R}$ и функций $f_{j} \geqslant 0, j \in \mathbb{N}$, а также равномерная аппроксимация п.п. по Степанову функций элементарными п.п. функциями [15]. Неотрицательность функций $f_{j}$ использовалась существенно, и предложенные в [14] доказательства не переносятся на общий случай.

В $\S 3$ определяются разные множества п.п. по Степанову мерозначных функций и устанавливаются соотношения между ними. В обшем случае условия $\mu[\cdot ; \cdot] \in S_{p}(\mathbb{R}, \mathscr{M}(U))$ и $\mu[\cdot ; \cdot] \in S_{p}^{w}(\mathbb{R}, \mathscr{M}(U))$ независимы. Выделяется множество $\widetilde{S}_{p}^{w}(\mathbb{R}, \mathscr{M}(U))$ мерозначных функций $\mathbb{R} \ni t \rightarrow \mu[\cdot ; t] \in \mathscr{M}(U)$ таких, что для любой периодической функции $\mathbb{R} \ni t \rightarrow F(\cdot, t) \in C_{b}(U)$, для которой $F(x, \cdot)$ - измеримая функция при всех $x \in U$ и $\operatorname{ess}^{\sup _{t \in \mathbb{R}}}\|F(\cdot, t)\|_{C_{b}(U)}<+\infty$, функция $t \rightarrow \int_{U} F(x, t) \mu[d x ; t]$ п.п. по Степанову (степени $p$ ). Для функций 
$\mu[\cdot ; \cdot] \in \widetilde{S}_{p}^{w}(\mathbb{R}, \mathscr{M}(U))$ справедливы утверждения, аналогичные утверждениям для п.п. по Бору мерозначных функций. Если $\mu[\cdot ; \cdot] \in S_{p}^{w}(\mathbb{R}, \mathscr{M}(U))$ и $\mu[\cdot ; t] \in \mathscr{M}^{+}(U)$ п.в., то $\mu[\cdot ; \cdot] \in \widetilde{S}_{p}^{w}(\mathbb{R}, \mathscr{M}(U))$.

В последних двух параграфах доказываются приведенные в предыдущих разделах теоремы 2 и 4 .

\section{§1. Определения, обозначения и некоторые утверждения}

Пусть $(U, \rho)$ - метрическое пространство. Функция $f: T \rightarrow U$, где $T \subset \mathbb{R}$ - измеримое (по Лебегу) множество, называется әлементарной, если найдутся измеримые (по Лебегу) непересекающиеся множества $T_{j} \subset T$ и точки $x_{j} \in U$ (множество индексов не более чем счетно) такие, что $m\left(T \backslash \bigcup T_{j}\right)=0(m(\cdot)-$ мера Лебега) и $f(t)=x_{j}$ при всех $t \in T_{j}$. Функция $f: T \rightarrow U$ (определенная при почти всех (п.в.) $t \in T$ ) измерима, если для любого $\varepsilon>0$ найдется элементарная функция $f_{\varepsilon}: T \rightarrow U$ такая, что $\rho\left(f(t), f_{\varepsilon}(t)\right)<\varepsilon$ при п.в. $t \in T$.

Зафиксируем точку $x_{0} \in U$. Через $M_{p}(\mathbb{R}, U), p \geqslant 1$, обозначим множество измеримых функций $f: \mathbb{R} \rightarrow U$ (совпадающие почти всюду (п.в.) функции отождествляются) таких, что

$$
\sup _{t \in \mathbb{R}} \int_{t}^{t+1} \rho^{p}\left(f(t), x_{0}\right) d t<+\infty .
$$

На $M_{p}(\mathbb{R}, U)$ вводится метрика

$$
D_{p}^{(\rho)}\left(f_{1}, f_{2}\right)=\sup _{t \in \mathbb{R}}\left(\int_{t}^{t+1} \rho^{p}\left(f_{1}(t), f_{2}(t)\right) d t\right)^{1 / p}, \quad f_{1}, f_{2} \in M_{p}(\mathbb{R}, U) .
$$

Множество функций $f \in M_{p}(\mathbb{R}, U)$, для которых

$$
\lim _{\delta \rightarrow+0} \sup _{t \in \mathbb{R}} \sup _{L \subset[t, t+1]: m(L) \leqslant \delta} \int_{L} \rho^{p}\left(f(t), x_{0}\right) d t=0,
$$

обозначим через $M_{p}^{\prime}(\mathbb{R}, U)$.

Для любого $\varepsilon>0$ число $\tau \in \mathbb{R}$ называется $\varepsilon$-почти периодом функции $f \in$ $M_{p}(\mathbb{R}, U)$, если $D_{p}^{(\rho)}(f(\cdot), f(\cdot+\tau))<\varepsilon$. Функция $f \in M_{p}(\mathbb{R}, U)$ принадлежит множеству $S_{p}(\mathbb{R}, U)$ почти периодических (п.п.) по Степанову степени $p \geqslant 1$ функций, если для любого $\varepsilon>0$ множество $\varepsilon$-почти периодов функции $f$ относительно плотно [11]. Справедливо вложение $S_{p}(\mathbb{R}, U) \subset M_{p}^{\prime}(\mathbb{R}, U)$. Последовательность $\left\{\tau_{j}\right\} \subset \mathbb{R}$ называется $f$-возвращающей для функции $f \in M_{p}(\mathbb{R}, U)$, если $D_{p}^{(\rho)}\left(f(\cdot), f\left(\cdot+\tau_{j}\right)\right) \rightarrow 0$ при $j \rightarrow+\infty$. Каждой функции $f \in S_{p}(\mathbb{R}, U)$ ставится в соответствие модуль (группа по сложению) $\operatorname{Mod} f \subset \mathbb{R}$, состоящий из тех чисел $\lambda \in \mathbb{R}$, для которых $e^{i \lambda \tau_{j}} \rightarrow 1\left(i^{2}=-1\right)$ при $j \rightarrow+\infty$ для любой $f$-возвращающей последовательности $\left\{\tau_{j}\right\}$. Если функция $f \in S_{p}(\mathbb{R}, U)$ не является (п.в.) постоянной, то Mod $f$-счетное множество.

На множестве $L_{\infty}(\mathbb{R}, U)$ измеримых и в сушественном ограниченных функций $f: \mathbb{R} \rightarrow U$ рассматривается метрика

$$
D_{\infty}^{(\rho)}\left(f_{1}, f_{2}\right)=\underset{t \in \mathbb{R}}{\operatorname{esssup}} \rho\left(f_{1}(t), f_{2}(t)\right), \quad f_{1}, f_{2} \in L_{\infty}(\mathbb{R}, U)
$$


Число $\tau \in \mathbb{R}$ называется $\varepsilon$-почти периодом функции $f \in L_{\infty}(\mathbb{R}, U)$, если $D_{\infty}^{(\rho)}(f(\cdot), f(\cdot+\tau))<\varepsilon$. Непрерывная ограниченная функция $f: \mathbb{R} \rightarrow U$ называется почти периодической по Бору (равномерной п.п.), если для любого $\varepsilon>0$ относительно плотно множество $\varepsilon$-почти периодов функции $f[11]$. Множество п.п. по Бору функций обозначим через $C A P(\mathbb{R}, U)$. Любая функция $f \in C A P(\mathbb{R}, U)$ равномерно непрерывна и $C A P(\mathbb{R}, U) \subset S_{p}(\mathbb{R}, U) \subset S_{1}(\mathbb{R}, U)$. Для функции $f \in C A P(\mathbb{R}, U)$ с помощью метрики $D_{\infty}^{(\rho)}\left(\right.$ как и для функций из $S_{p}(\mathbb{R}, U)$ ) определяются $f$-возвращающие последовательности $\left\{\tau_{j}\right\}$ (и $\left.\operatorname{Mod} f\right)$. При этом $f$-возврашаюшие последовательности для п.п. функций $f$ не зависят от того, какому из рассматриваемых пространств их считать принадлежащими.

Если $f \in C A P(\mathbb{R}, U)$, то $\{f(t): t \in \mathbb{R}\} \subset U$ - вполне ограниченное множество. Если $f \in S_{p}(\mathbb{R}, U), p \geqslant 1$, то для любого $\varepsilon>0$ существует вполне ограниченное множество $K_{\varepsilon} \subset U$ такое, что $\sup _{t \in \mathbb{R}} m\left(\left\{\xi \in[t, t+1]: f(\xi) \notin K_{\varepsilon}\right\}\right)<\varepsilon$.

В случае $U=\mathbb{R}$ будут использоваться краткие обозначения $M_{p}(\mathbb{R}), M_{p}^{\prime}(\mathbb{R})$, $S_{p}(\mathbb{R})$ и $C A P(\mathbb{R})$. Для измеримого множества $T \subset \mathbb{R}$ обычным образом определяются пространства $L_{p}(T, U), p \geqslant 1$, и $L_{\infty}(T, U)$.

Для модулей $\Lambda_{j} \subset \mathbb{R}$ через $\sum_{j} \Lambda_{j}$ обозначается наименшший модуль (группа по сложению), содержащий все множества $\Lambda_{j}$.

Если $f, f_{i} \in S_{p}(\mathbb{R}, U), i \in \mathbb{N}$, то $\operatorname{Mod} f \subset \sum_{i} \operatorname{Mod} f_{i}$ в том и только том случае,

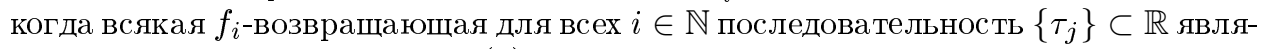
ется $f$-возврашаюшей. Если $D_{p}^{(\rho)}\left(f, f_{i}\right) \rightarrow 0$ при $i \rightarrow+\infty$, то $\operatorname{Mod} f \subset \sum_{i} \operatorname{Mod} f_{i}$.

Пусть $C_{b}(U)$ - банахово пространство непрерывных ограниченных функций $F: U \rightarrow \mathbb{R}$ с нормой $\|F\|_{C_{b}(U)}=\sup _{x \in U}|F(x)|, C_{b}^{+}(U)-$ множество неотрицательных функций из $C_{b}(U), \mathscr{L}(U)=\left\{F \in C_{b}(U): 0 \leqslant F(x) \leqslant 1\right.$ и $|F(x)-F(y)| \leqslant \rho(x, y)$ для всех $x, y \in U\}, F\left(\left.\cdot\right|_{A}\right)$ - сужение функции $F: U \rightarrow \mathbb{R}$ на множество $A \subset U$. Для любых функции $F \in C_{b}(U)$, числа $\varepsilon>0$ и компакта $K \subset U$ существует функция $G \in C_{b}(U)$ такая, что $\lambda G(\cdot)+\gamma \in \mathscr{L}(U)$ для некоторых чисел $\lambda>0$ и $\gamma \in \mathbb{R}, \inf _{y \in U} F(y) \leqslant G(x) \leqslant \sup _{y \in U} F(y)$ для всех $x \in U$ и $|F(x)-G(x)|<\varepsilon$ для всех $x \in K$.

Будем далее предполагать, что пространство $(U, \rho)$ полное и сепарабельное. Пусть $\mathscr{B}(U)$ - множество борелевских подмножеств пространства $U$ (наименьшая $\sigma$-алгебра, содержащая открытые множества), $\mathscr{M}(U)$ - линейное пространство борелевских знакопеременных мер (зарядов) $\mu: \mathscr{B}(U) \rightarrow \mathbb{R}, \mathscr{M}^{+}(U)$ - множество неотрицательных мер $\mu \in \mathscr{M}(U)$. Через $\mu[\mathscr{O}]$ будем обозначать значение меры $\mu \in \mathscr{M}(U)$ на множестве $\mathscr{O} \in \mathscr{B}(U)$ и через $\mu(F)$ значение линейного непрерьвного функционала

$$
C_{b}(U) \ni F \rightarrow \mu(F)=\int_{U} F(x) \mu[d x],
$$

$\delta_{x}$ - мера Дирака, сосредоточенная в точке $x \in U$. Для мер $\mu \in \mathscr{M}(U)$ справедливо представление $\mu=\mu^{+}-\mu^{-}$, где $\mu^{ \pm} \in \mathscr{M}^{+}(U)$ и $\mu^{+}\left[\mathscr{O}_{\mu}\right]=\mu^{-}\left[U \backslash \mathscr{O}_{\mu}\right]=0$ для некоторого множества $\mathscr{O}_{\mu} \in \mathscr{B}(U) ;|\mu|=\mu^{+}+\mu^{-},|\mu|[U]=\sup \left\{\mu(F): F \in C_{b}(U)\right.$, $\left.\|F\|_{C_{b}(U)} \leqslant 1\right\}$. Каждая мера $\mu \in \mathscr{M}(U)$ является радоновской, т.е. для любых $\mathscr{O} \in \mathscr{B}(U)$ и $\varepsilon>0$ существует компакт $K \subset \mathscr{O}$ такой, что $|\mu|[\mathscr{O} \backslash K]<\varepsilon$. На линейном пространстве $\mathscr{M}(U)$ определим норму

$$
\|\mu\|_{w}=\sup _{F \in \mathscr{L}(U)}|\mu(F)|, \quad \mu \in \mathscr{M}(U) .
$$


Пространство $\left(\mathscr{M}(U),\|\cdot\|_{w}\right)$ - сепарабельное и является полным тогда и только тогда, когда найдется число $\varepsilon>0$ такое, что $\rho(x, y) \geqslant \varepsilon$ для всех $x, y \in U$, $x \neq y$. Множество $\mathscr{M}^{+}(U)$ (в отличие от пространства $\mathscr{M}(U)$ ) всегда полное. Для любых числа $b \geqslant 0$ и семейства компактов $K_{\delta} \subset U, \delta>0$, обозначим $\mathscr{K}\left(b,\left\{K_{\delta}\right\}\right)=\left\{\mu \in \mathscr{M}(U):|\mu|[U] \leqslant b\right.$ и $|\mu|\left[U \backslash K_{\delta}\right] \leqslant \delta$ для всех $\left.\delta>0\right\}$. Множества $\mathscr{K}\left(b,\left\{K_{\delta}\right\}\right) \subset\left(\mathscr{M}(U),\|\cdot\|_{w}\right)$ выпуклые и компактные, $\mathscr{K}_{U}$ - совокупность всех множеств $\mathscr{K}\left(b,\left\{K_{\delta}\right\}\right)$.

Если $K \subset \mathscr{M}^{+}(U)$ и $K-$ компактное множество, то $K \subset A$ для некоторого множества $A \in \mathscr{K}_{U}$. Если $\mu, \mu_{j} \in A \in \mathscr{K}_{U}, j \in \mathbb{N}$, то $\left\|\mu-\mu_{j}\right\|_{w} \rightarrow 0$ при $j \rightarrow+\infty$ тогда и только тогда, когда $\mu_{j}(F) \rightarrow \mu(F)$ при $j \rightarrow+\infty$ для всех функций $F \in C_{b}(U)$. Поэтому если $A \in \mathscr{K}_{U}$ или $A=\mathscr{M}^{+}(U)$, то для любой функции $F \in C_{b}(U)$ функция $A \ni \mu \rightarrow \mu(F) \in \mathbb{R}$ непрерывна.

Лемма 1. Если $\left(V, \rho_{V}\right)$ - полное метрическое пространство, $f \in C A P(\mathbb{R}, V)$ и функиия $\mathbb{R} \ni t \rightarrow F(\cdot, t) \in C_{b}(V)$ принадлежст пространству $C A P\left(\mathbb{R}, C_{b}(V)\right)$, mo $F(f(\cdot), \cdot) \in C A P(\mathbb{R}) u \operatorname{Mod} F(f(\cdot), \cdot) \subset \operatorname{Mod} f(\cdot)+\operatorname{Mod} F(\cdot, \cdot)$.

СлЕДСТВИЕ. Пусть $(U, \rho)$ - полное сепарабельное метрическое пространство, функиия $\mathbb{R} \ni t \rightarrow \mu[\cdot ; t] \in A \in \mathscr{K}_{U}$ принадлежит пространству $C A P\left(\mathbb{R},\left(\mathscr{M}(U),\|\cdot\|_{w}\right)\right)$ u $F(\cdot, \cdot) \in C A P\left(\mathbb{R}, C_{b}(U)\right)$. Tогда $\mu(F(\cdot, \cdot) ; \cdot) \in C A P(\mathbb{R})$ $u \operatorname{Mod} \mu(F(\cdot, \cdot) ; \cdot) \subset \operatorname{Mod} \mu[\cdot ; \cdot]+\operatorname{Mod} F(\cdot, \cdot)$.

Лемма 2. Пусть $\left(V, \rho_{V}\right)$ - полное метрическое пространство, $f \in S_{p}(\mathbb{R}, V)$, $p \geqslant 1, V \ni x \rightarrow F(x, t) \in \mathbb{R}, t \in \mathbb{R},-$ непрерьвнье функции такие, что $|F(x, t)| \leqslant a_{1} \rho\left(x, x_{0}\right)+a_{2}$ для всех $x \in V, t \in \mathbb{R}$, где $a_{1}, a_{2} \geqslant 0$, $x_{0} \in V, u F\left(\left.\cdot\right|_{K}, \cdot\right) \in S_{1}\left(\mathbb{R}, C_{b}(K)\right)$ для всех (непустых) компактных множсеств $K \subset V$. Tогда $F(f(\cdot), \cdot) \in S_{p}(\mathbb{R}) u \operatorname{Mod} F(f(\cdot), \cdot) \subset \operatorname{Mod} f(\cdot)+$ $\sum_{j} \operatorname{Mod} F\left(\left.\cdot\right|_{K_{j}}, \cdot\right)$, әде $K_{j} \subset V, j \in \mathbb{N}$, - любие компактние множества, для которых $\lim _{j \rightarrow+\infty} \sup _{t \in \mathbb{R}} m\left(\left\{\xi \in[t, t+1]: f(\xi) \notin \bigcup_{i \leqslant j} K_{i}\right\}\right)=0$.

Лемма 1 хорошо известна (см., например, [16]). Лемма 2 непосредственно следует из результатов работ [15] и [17].

Для произвольной функции $\mathbb{R} \ni t \rightarrow \mu[\cdot ; t] \in \mathscr{M}(U)$ следующие условия эквивалентны:

1) $t \rightarrow \mu[\mathscr{O} ; t]-$ измеримая функция для любого множества $\mathscr{O} \in \mathscr{B}(U)$;

2) $t \rightarrow \mu(F ; t)$ - измеримая функция для любой функции $F \in C_{b}(U)$;

3) $t \rightarrow \mu[\cdot ; t] \in\left(\mathscr{M}(U),\|\cdot\|_{w}\right)$ - измеримая функция.

Пусть $\mathbb{R} \ni t \rightarrow \mu[\cdot ; t] \in \mathscr{M}(U)$ - измеримая функция. Тогда для любого $\varepsilon>0$ и любого отрезка $[a, b], a \leqslant b$, найдутся измеримое множество $T \subset[a, b]$ и множество $A \in \mathscr{K}_{U}$ такие, что $m([a, b] \backslash T)<\varepsilon$ и $\mu[\cdot ; t] \in A$ для всех $t \in T$. Функция $t \rightarrow$ $|\mu|[\cdot ; t]$ также измерима, и для любого $\varepsilon>0$ существует элементарная функция $\mathbb{R} \ni t \rightarrow F(\cdot, t) \in C_{b}(U)$, для которой $\|F(\cdot, t)\|_{C_{b}(U)} \leqslant 1$ и $\mu(F(\cdot, t) ; t) \geqslant$ $|\mu|[U ; t]-\varepsilon$ при п.в. $t \in \mathbb{R}$.

\section{§2. Мерозначные слабо почти периодические по Бору функции}

Через $C A P^{w}(\mathbb{R}, \mathscr{M}(U))$ обозначается множество слабо п.п. по Бору мерозначных функций $\mathbb{R} \ni t \rightarrow \mu[\cdot ; t] \in \mathscr{M}(U)$ (таких, что $\mu(F ; \cdot) \in C A P(\mathbb{R})$ для всех $\left.F \in C_{b}(U)\right)$. 
ТЕОРЕМа 1. Пусть $(U, \rho)$ - полное сепарабельное метрическое пространство $и \mathbb{R} \ni t \rightarrow \mu[\cdot ; t] \in \mathscr{M}(U)-$ произвольная мерозначная функиия. Тогда следующие условия эквивалентны:

1) $\mu[\cdot ; \cdot] \in C A P^{w}(\mathbb{R}, \mathscr{M}(U))$;

2) $\mu[\cdot ; \cdot] \in C A P\left(\mathbb{R},\left(\mathscr{M}(U),\|\cdot\|_{w}\right)\right)$ и найдется множсество $A \in \mathscr{K}_{U}$ такое, что $\mu[\cdot ; t] \in A$ для всех $t \in \mathbb{R}$.

Если выполнены условия 1) и 2), то

$$
\operatorname{Mod} \mu[\cdot ; \cdot]=\sum_{F \in C_{b}(U)} \operatorname{Mod} \mu(F ; \cdot)
$$

При доказательстве теоремы 1 используются лемма 3 и теорема 2. Доказательство теоремы 2 приведено в $\S 4$.

Если $\mu[\cdot ; \cdot] \in C A P\left(\mathbb{R},\left(\mathscr{M}(U),\|\cdot\|_{w}\right)\right)$, то не всегда $\mu[\cdot ; t] \in A$ при всех $t \in \mathbb{R}$, где $A$ - некоторое множество из $\mathscr{K}_{U}$. Действительно, пусть $U=[-1,1], q>1$, $\mu[\cdot ; t]=|\sin t|^{-1 / q}\left(\delta_{\sin t}[\cdot]-\delta_{0}[\cdot]\right)$ при $t \neq n \pi, n \in \mathbb{Z}$, и $\mu[\cdot ; t]=0$ в противном случае. Тогда $t \rightarrow \mu[\cdot ; t]$ - непрерьвная периодическая с периодом $2 \pi$ функция и $\int_{0}^{2 \pi}(|\mu|[U ; t])^{q} d t=+\infty$.

Если $\mu \in C A P(\mathbb{R}, \mathscr{M}(U))$ и $\mu[\cdot ; t] \in \mathscr{M}^{+}(U), t \in \mathbb{R}$, то существует множество $A \in \mathscr{K}_{U}$ такое, что $\mu[\cdot ; t] \in A$ при всех $t \in \mathbb{R}$.

Через $\bar{A}$ обозначается замыкание множества $A \subset U, \rho(x, A)=\inf _{y \in A} \rho(x, y)$, $\rho(x, \varnothing)=1, x \in U ; \rho(A, B)=\inf _{x \in A, y \in B} \rho(x, y), \rho(A, \varnothing)=1, B \subset U ; A^{\delta}=$ $\{x \in U: \rho(x, A)<\delta\}, \delta>0, \varnothing^{\delta}=\varnothing ; \operatorname{supp} F=\overline{\{x \in U: F(x) \neq 0\}}, F \in C_{b}(U)$.

Если $F_{j} \in C_{b}^{+}(U), j \in \mathbb{N}$, и $F_{j}(x) \downarrow 0$ при $j \rightarrow+\infty\left(F_{j}(x) \geqslant F_{j+1}(x), j \in \mathbb{N}\right.$, и $F_{j}(x) \rightarrow 0$ при $\left.j \rightarrow+\infty\right)$ для всех $x \in U$, то $\max _{x \in K} F_{j}(x) \rightarrow 0$ при $j \rightarrow+\infty$ для любого (непустого) компакта $K \subset U$. Поэтому $\max _{\mu \in A}\left|\mu\left(F_{j}\right)\right| \rightarrow 0$ при $j \rightarrow+\infty$ для любого множества $A \in \mathscr{K}_{U}$.

Лемма 3. Пусть $B \subset \mathscr{M}(U)$ - непустое множество такое, что

$$
\sup _{\mu \in B}|\mu(F)|<+\infty
$$

для всех $F \in C_{b}(U)$ и для любой последовательности функиий $F_{j} \in C_{b}^{+}(U)$, $j \in \mathbb{N}$, для которой $F_{j}(x) \downarrow 0, j \rightarrow+\infty$, при всех $x \in U$, имеем

$$
\sup _{\mu \in B}\left|\mu\left(F_{j}\right)\right| \rightarrow 0, \quad j \rightarrow+\infty
$$

Тогда $B \subset A$ для некоторого множества $A \in \mathscr{K}_{U}$.

ДокАЗАтЕльство. Можно считать, что множество $B$ содержит вместе с каждой мерой $\mu$ также и меру $-\mu$. Существование числа $b \geqslant 0$ такого, что $|\mu|[U] \leqslant b$ для всех мер $\mu \in B$, следует из условия леммы и принципа равномерной ограниченности. Покажем, что для любых $\varepsilon, \delta>0$ существует компакт $K_{\varepsilon, \delta} \subset U$ такой, что $|\mu|\left[U \backslash K_{\varepsilon, \delta}^{\delta}\right]<\varepsilon$ для всех $\mu \in B$. Допустим противное. Тогда найдутся числа $\varepsilon, \delta>0$ такие, что для любого компакта $K \subset U$ сушествует мера $\mu \in B$, для 
которой $|\mu|\left[U \backslash K^{\delta}\right] \geqslant \varepsilon$. В этом случае можно выбрать меры $\mu_{j} \in B$ и компакты $K_{j} \subset U, j \in \mathbb{N}$, такие, что $K_{j} \subset K_{j+1},\left|\mu_{i}\right|\left[U \backslash K_{j}\right]<2^{i-j-4} \varepsilon, i=1, \ldots, j$, и $\left|\mu_{j+1}\right|\left[U \backslash K_{j}^{\delta}\right] \geqslant \varepsilon$ для всех $j \in \mathbb{N}$. Обозначим $X_{j}=K_{j+1} \backslash K_{j}^{\delta}$. Имеем $\left|\mu_{j+1}\right|\left[X_{j}\right] \geqslant$ $\left|\mu_{j+1}\right|\left[U \backslash K_{j}^{\delta}\right]-\left|\mu_{j+1}\right|\left[U \backslash K_{j+1}\right]>\varepsilon-\varepsilon / 16, j \in \mathbb{N}$. Изменяя в случае необходимости знак у мер $\mu_{j+1}$, можно считать, что $\mu_{j+1}^{+}\left[X_{j}\right] \geqslant \mu_{j+1}^{-}\left[X_{j}\right]$. Найдутся компакты $K_{j}^{ \pm} \subset X_{j}, j \in \mathbb{N}$, такие, что $K_{j}^{+} \cap K_{j}^{-}=\varnothing, \mu_{j+1}^{ \pm}\left[K_{j}^{ \pm}\right]>\mu_{j+1}^{ \pm}\left[X_{j}\right]-\varepsilon / 8$, $\mu_{j+1}^{ \pm}\left[K_{j}^{\mp}\right]=0$. Для всех $i, j \in \mathbb{N}, i \neq j$, справедливы неравенства $\rho\left(X_{i}, X_{j}\right) \geqslant \delta$. Выберем числа $\delta_{j} \in(0, \delta / 3], j \in \mathbb{N}$, так, что $\left|\mu_{i}\right|\left[X_{j}^{\delta_{j}} \backslash X_{j}\right]<2^{i-j-6} \varepsilon$ при всех $i=1, \ldots, j+1$. Пусть $G_{j} \in C_{b}^{+}(U), j \in \mathbb{N},-$ функции, для которых $0 \leqslant G_{j}(x) \leqslant 1$ при всех $x \in U, G_{j}(x)=1$ при $x \in K_{j}^{+}$и $G_{j}(x)=0$ при $x \in K_{j}^{-} \cup\left(U \backslash X_{j}^{\delta_{j}}\right)$. Имеем

$$
\begin{aligned}
\mu_{j+1}\left(G_{j}\right) & \geqslant \mu_{j+1}^{+}\left[K_{j}^{+}\right]-\mu_{j+1}^{-}\left[X_{j} \backslash K_{j}^{-}\right]-\left|\mu_{j+1}\right|\left[X_{j}^{\delta_{j}} \backslash X_{j}\right] \\
& >\mu_{j+1}^{+}\left[K_{j}^{+}\right]-\frac{\varepsilon}{8}-\frac{\varepsilon}{32}>\frac{1}{2}\left|\mu_{j+1}\right|\left[X_{j}\right]-\frac{\varepsilon}{4}-\frac{\varepsilon}{32}>\frac{3 \varepsilon}{16}
\end{aligned}
$$

для всех $j \in \mathbb{N}$. При этом $\rho\left(\operatorname{supp} G_{i}, \operatorname{supp} G_{j}\right) \geqslant \delta / 3$ для всех $i, j \in \mathbb{N}, i \neq j$. Определим функции $F_{j}(x)=\sum_{i \geqslant j} G_{i}(x), x \in U, j \in \mathbb{N} ; F_{j} \in C_{b}^{+}(U)$ и $F_{j}(x) \downarrow 0$ при $j \rightarrow+\infty$ для всех $x \in U$. Поэтому $\sup _{\mu \in B}\left|\mu\left(F_{j}\right)\right| \rightarrow 0$ при $j \rightarrow+\infty$. С другой стороны, для всех $j \in \mathbb{N}$ имеем $\mu_{j+1}\left(F_{j}\right)=\mu_{j+1}\left(G_{j}\right)+\sum_{i \geqslant j+1} \mu_{j+1}\left(G_{i}\right)$, при этом $\mu_{j+1}\left(G_{j}\right)>3 \varepsilon / 16$ и

$\left|\mu_{j+1}\left(G_{i}\right)\right| \leqslant\left|\mu_{j+1}\right|\left[X_{i}^{\delta_{i}} \backslash X_{i}\right]+\left|\mu_{j+1}\right|\left[X_{i}\right]<2^{j-i-5} \varepsilon+\left|\mu_{j+1}\right|\left[U \backslash K_{i}\right]<5 \cdot 2^{j-i-5} \varepsilon$,

$i \geqslant j+1$. Следовательно, $\mu_{j+1}\left(F_{j}\right)>\varepsilon / 32, j \in \mathbb{N}$. Полученное противоречие доказывает сушествование требуемого компакта $K_{\varepsilon, \delta} \subset U$. Зафиксируем $\varepsilon>0$. Найдем компакты $K_{j} \subset U, j \in \mathbb{N}$, такие, что $|\mu|\left[U \backslash K_{j}^{1 / j}\right]<2^{-j} \varepsilon$ для всех мер $\mu \in B$. Положим $K=\bigcap_{j} \overline{K_{j}^{1 / j}}$. Множество $K$ компактное и

$$
|\mu|[U \backslash K] \leqslant \sum_{j}|\mu|\left[U \backslash K_{j}^{1 / j}\right]<\varepsilon
$$

для всех мер $\mu \in B$. Лемма доказана.

Пусть $(\mathscr{H},\|\cdot\|)-\left(\right.$ вещественное или комплексное) банахово пространство, $\mathscr{S}_{l}$ - совокупность непустых подмножеств множества $\{j \in \mathbb{N}: j \geqslant l\}, \mathscr{S}_{l}^{\prime}$ - совокупность конечных подмножеств из $\mathscr{S}_{l}, l \in \mathbb{N}$. Множество последовательностей $\left\{x_{j}\right\}_{j \in \mathbb{N}} \subset \mathscr{H}$, для которых ряд $\sum_{j \in \mathbb{N}} x_{j}$ безусловно (при любом порядке суммирования) сходится, обозначим через $Q \mathscr{H}$. Сумма безусловно сходяшегося ряда не зависит от порядка суммирования.

Пусть $\left\{x_{j}\right\}_{j \in \mathbb{N}} \subset \mathscr{H}$. Обозначим $N_{l}\left[\left\{x_{j}\right\}\right]=\sup _{J \in \mathscr{S}_{l}^{\prime}}\left\|\sum_{j \in J} x_{j}\right\|$ (возможно, $\left.N_{l}\left[\left\{x_{j}\right\}\right]=+\infty\right), l \in \mathbb{N}$. Справедливы неравенства $N_{l}\left[\left\{x_{j}\right\}\right] \geqslant N_{l+1}\left[\left\{x_{j}\right\}\right], l \in \mathbb{N}$. Для множества $J \in \mathscr{S}_{1}$ ряд $\sum_{j \in J} x_{j}$ сходится, если сушествует

$$
\lim _{l \rightarrow+\infty} \sum_{j \in J, j \leqslant l} x_{j}=\sum_{j \in J} x_{j} .
$$


Следующие условия эквивалентны:

1) $\left\{x_{j}\right\} \in Q \mathscr{H}$;

2) для любого непустого множества $J \subset \mathbb{N}$ ряд $\sum_{j \in J} x_{j}$ сходится;

3) $N_{1}\left[\left\{x_{j}\right\}\right]<+\infty$ и $N_{l}\left[\left\{x_{j}\right\}\right] \rightarrow 0$ при $l \rightarrow+\infty$.

Для непустого множества $T$ обозначим через $Q \mathscr{H}(T)$ множество последовательностей $\left\{f_{j}\right\}$ функций $f_{j}: T \rightarrow \mathscr{H}, j \in \mathbb{N}$, таких, что $\left\{f_{j}(t)\right\} \in Q \mathscr{H}$ для всех $t \in T$. Для $\left\{f_{j}\right\} \in Q_{\mathscr{H}}(T)$ и $J \in \mathscr{S}_{1}$ положим $f(J ; t)=\sum_{j \in J} f_{j}(t), t \in T$; $f(\{j\} ; \cdot)=f_{j}(\cdot), j \in \mathbb{N}, f(\varnothing ; t)=0, t \in T$.

Tеорема 2. Пусть $(\mathscr{H},\|\cdot\|)$ - (вещественное или комплексное) банахово пространство и $\left\{f_{j}\right\} \in Q_{\mathscr{H}}(\mathbb{R})$. Предположим, ито $f(J ; \cdot) \in C A P(\mathbb{R}, \mathscr{H})$ для всех непустых множеств $J \subset \mathbb{N}$. Тогда $\sup _{t \in \mathbb{R}} N_{l}\left[\left\{f_{j}(t)\right\}\right] \rightarrow 0$ при $l \rightarrow+\infty$.

ДОКАЗАТЕЛЬСТВо ТЕОРЕМЫ 1. ИМпликация 2) $\Rightarrow 1$ ) и вложение

$$
\sum_{F \in C_{b}(U)} \operatorname{Mod} \mu(F ; \cdot) \subset \operatorname{Mod} \mu[\cdot ; \cdot]
$$

вытекают из следствия леммы 1 . Докажем, что из 1$)$ следует 2). Пусть $\mu[\cdot ; \cdot] \in$ $C A P^{w}(\mathbb{R}, \mathscr{M}(U)), F_{j} \in C_{b}^{+}(U), j \in \mathbb{N}$, и $F_{j}(x) \downarrow 0$ при $j \rightarrow+\infty$ для всех $x \in U$. Обозначим $f_{j}(t)=\mu\left(F_{j}-F_{j+1} ; t\right), j \in \mathbb{N}, t \in \mathbb{R}$. Так как для всех непустых множеств $J \subset \mathbb{N}$ функция $U \ni x \rightarrow \sum_{j \in J}\left(F_{j}(x)-F_{j+1}(x)\right)$ непрерывна (и ограничена), то $f(J ; \cdot) \in C A P(\mathbb{R})$. С другой стороны, для всех $l \in \mathbb{N}$ имеем $\sup _{t \in \mathbb{R}}\left|\mu\left(F_{l} ; t\right)\right| \leqslant \sup _{t \in \mathbb{R}} N_{l}\left[\left\{f_{j}(t)\right\}\right]$. Поэтому из теоремы 2 получаем $\sup _{t \in \mathbb{R}}\left|\mu\left(F_{l} ; t\right)\right| \rightarrow 0$ при $l \rightarrow+\infty$. Из леммы 3 вытекает существование множества $\mathscr{K}\left(b,\left\{K_{\delta}\right\}\right) \in \mathscr{K}_{U}$ такого, что $\mu[\cdot ; t] \in \mathscr{K}\left(b,\left\{K_{\delta}\right\}\right)$ при всех $t \in \mathbb{R}$.

Зафиксируем $\varepsilon>0$. Выберем любое число $\varepsilon^{\prime}>0: \varepsilon^{\prime} b<\varepsilon$. Множество $\mathscr{L}\left(K_{\varepsilon}\right)=\left\{F\left(\left.\cdot\right|_{K_{\varepsilon}}\right): F \in \mathscr{L}(U)\right\} \subset C_{b}\left(K_{\varepsilon}\right)$ компактно, поэтому найдутся функции $F_{j}^{(\varepsilon)} \in \mathscr{L}(U), j=1, \ldots, n(\varepsilon)$, для которых множество $\left\{F_{j}^{(\varepsilon)}\left(\left.\cdot\right|_{K_{\varepsilon}}\right)\right\}_{j=1, \ldots, n(\varepsilon)}$ образует $\varepsilon^{\prime}$-сеть для $\mathscr{L}\left(K_{\varepsilon}\right)$. Для любой функции $F \in \mathscr{L}(U)$ найдется номер $j \in\{1, \ldots, n(\varepsilon)\}$ такой, что $\max _{x \in K_{\varepsilon}}\left|F(x)-F_{j}^{(\varepsilon)}(x)\right|<\varepsilon^{\prime}$. Следовательно, для всех $\mu \in \mathscr{K}\left(b,\left\{K_{\delta}\right\}\right)$ имеем

$$
\left|\mu(F)-\mu\left(F_{j}^{(\varepsilon)}\right)\right| \leqslant 2|\mu|\left[U \backslash K_{\varepsilon}\right]+\varepsilon^{\prime} b<3 \varepsilon .
$$

Если $\tau$ - любой общий $\varepsilon$-почти период функций $\mu\left(F_{j}^{(\varepsilon)} ; \cdot\right), j=1, \ldots, n(\varepsilon)$ (множество таких чисел относительно плотно [11]), то

$$
\begin{aligned}
\sup _{t \in \mathbb{R}}\|\mu[\cdot ; t]-\mu[\cdot ; t+\tau]\|_{w} & =\sup _{t \in \mathbb{R}} \sup _{F \in \mathscr{L}(U)}|\mu(F ; t)-\mu(F ; t+\tau)| \\
& \leqslant 6 \varepsilon+\sup _{t \in \mathbb{R}} \max _{j=1, \ldots, n(\varepsilon)}\left|\mu\left(F_{j}^{(\varepsilon)} ; t\right)-\mu\left(F_{j}^{(\varepsilon)} ; t+\tau\right)\right|<7 \varepsilon .
\end{aligned}
$$

Так как число $\varepsilon>0$ выбирается произвольно, то $\mu[\cdot ; \cdot] \in C A P(\mathbb{R}, \mathscr{M}(U))$. Пусть $\varepsilon_{i} \rightarrow+0$ при $i \rightarrow+\infty$ и $\left\{\tau_{l}\right\}$ есть $\mu\left(F_{j}^{\left(\varepsilon_{i}\right)} ; \cdot\right)$-возврашаюшая для всех $i \in \mathbb{N}$ и $j=1, \ldots, n\left(\varepsilon_{i}\right)$ последовательность. Тогда из (2) следует, что $\left\{\tau_{l}\right\}-$ также $\mu[\cdot ; \cdot]$-возврашаюшая последовательность. Поэтому

$$
\operatorname{Mod} \mu[\cdot ; \cdot] \subset \sum_{i, j} \operatorname{Mod} \mu\left(F_{j}^{\left(\varepsilon_{i}\right)} ; \cdot\right) \subset \sum_{F \in C_{b}(U)} \operatorname{Mod} \mu(F ; \cdot) .
$$

Вместе с обратным вложением это доказывает равенство (1). Теорема доказана. 


\section{§ 3. Мерозначные слабо почти периодические по Степанову функции}

Пусть $(U, \rho)$ - полное сепарабельное метрическое пространство, $L_{\infty}^{\circ}\left(\mathbb{R}, C_{b}(U)\right)$ - банахово пространство (определенных при п.в. $t \in \mathbb{R}$ ) в сушественном ограниченных функций $\mathbb{R} \ni t \rightarrow F(\cdot, t) \in C_{b}(U)$ (отождествляются функции, которые при п.в. $t \in \mathbb{R}$ совпадают) таких, что для любого $x \in U$ функция $\mathbb{R} \ni t \rightarrow$ $F(x, t) \in \mathbb{R}$ измерима (тогда для любого непустого компакта $K \subset U$ измерима функция $\left.\mathbb{R} \ni t \rightarrow F\left(\left.\cdot\right|_{K}, t\right) \in\left(C_{b}(K),\|\cdot\|_{C_{b}(K)}\right)\right)$. На $L_{\infty}^{\circ}\left(\mathbb{R}, C_{b}(U)\right)$ pacсматривается норма $\|F\|_{\infty}=\operatorname{ess}_{\sup _{t \in \mathbb{R}}}\|F(\cdot, t)\|_{C_{b}(U)}, F \in L_{\infty}^{\circ}\left(\mathbb{R}, C_{b}(U)\right)$. Для любой измеримой мерозначной функции $t \rightarrow \mu[\cdot ; t] \in \mathscr{M}(U)$ и любой функции $F \in L_{\infty}^{\circ}\left(\mathbb{R}, C_{b}(U)\right)$ функция $t \rightarrow \mu(F(\cdot, t) ; t)$ также измерима. Обозначим через $\mathscr{F}_{U}$ множество линейных (замкнутых) подпространств $\Phi$ банахова пространства $L_{\infty}^{\circ}\left(\mathbb{R}, C_{b}(U)\right)$, для которых выполнены следуюшие условия:

1) $|F(\cdot, \cdot)| \in \Phi$ для всех функций $\mathbb{R} \ni t \rightarrow F(\cdot, t) \in C_{b}(U)$ из $\Phi ;$

2) $F_{1}(\cdot, \cdot) F_{2}(\cdot, \cdot) \in \Phi$ для всех функций $F_{i}(\cdot, \cdot) \in \Phi, i=1,2$;

3) подпространство $\Phi$ содержит все постоянные по $t$ функции $t \rightarrow F(\cdot, t)=$ $F(\cdot) \in C_{b}(U)$

4) для любой функции $F(\cdot, \cdot) \in \Phi$, любого непустого компакта $K \subset U$ и любого $\varepsilon>0$ найдутся измеримое множество $T \subset \mathbb{R}$ и компакт $\mathscr{C} \subset C_{b}(K)$ такие, что $\sup _{t \in \mathbb{R}} m([t, t+1] \backslash T)<\varepsilon$ и $F\left(\left.\cdot\right|_{K}, t\right) \in \mathscr{C}$ при всех $t \in T$;

5) если $F(\cdot, \cdot) \in L_{\infty}^{\circ}\left(\mathbb{R}, C_{b}(U)\right), F_{j}(\cdot, \cdot) \in \Phi, j \in \mathbb{N}$, при п.в. $t \in \mathbb{R}$ для любого непустого компакта $K \subset U \max _{x \in K}\left|F(x, t)-F_{j}(x, t)\right| \rightarrow 0, j \rightarrow+\infty$, при этом также $\left\|F\left(\left.\cdot\right|_{K}, \cdot\right)-F_{j}\left(\left.\cdot\right|_{K}, \cdot\right)\right\|_{M_{1}\left(\mathbb{R}, C_{b}(K)\right)} \rightarrow 0, j \rightarrow+\infty$ (для любого компакта $K \subset U, K \neq \varnothing)$, то $F(\cdot, \cdot) \in \Phi$.

Для подпространств $\Phi \in \mathscr{F}_{U}$ обозначим $\Phi^{+}=\{F \in \Phi:$ при п.в. $t \in \mathbb{R} F(x, t) \geqslant 0$ для всех $x \in U\}, B_{1}(\Phi)=\left\{F \in \Phi:\|F\|_{\infty} \leqslant 1\right\}$. Пусть $\Phi_{a p}=\{F(\cdot, \cdot) \in$ $L_{\infty}^{\circ}\left(\mathbb{R}, C_{b}(U)\right): F\left(\left.\cdot\right|_{K}, \cdot\right) \in S_{1}\left(\mathbb{R}, C_{b}(K)\right)$ для любого непустого компакта $\left.K \subset U\right\} ;$ $\Phi_{\tau}, \tau>0,-$ линейное подпространство пространства $L_{\infty}^{\circ}\left(\mathbb{R}, C_{b}(U)\right)$, состоящее из периодических по $t$ функций $\mathbb{R} \ni t \rightarrow F(\cdot, t) \in C_{b}(U)$ с периодом $\tau$; $\Phi_{0}-$ подпространство постоянных по $t$ функций $U \times \mathbb{R} \ni(x, t) \rightarrow F(x, t)=F(x)$, $F \in C_{b}(U)$. Подпространства $\Phi_{a p}, \Phi_{\tau}, \tau>0$, и $\Phi_{0}$ принадлежат $\mathscr{F}_{U}$.

Обозначим через $S_{p}^{(\Phi)}(\mathbb{R}, \mathscr{M}(U)), p \geqslant 1, \Phi \in \mathscr{F}_{U}$, множество (п.в. определенных) мерозначных функций $\mathbb{R} \ni t \rightarrow \mu[\cdot ; t] \in \mathscr{M}(U)$ таких, что $\mu(F(\cdot, \cdot) ; \cdot) \in S_{p}(\mathbb{R})$ для всех функций $F(\cdot, \cdot) \in \Phi$. Мерозначные функции $\mu[\cdot ; \cdot] \in S_{p}^{(\Phi)}(\mathbb{R}, \mathscr{M}(U))$ измеримы. В дальнейшем будут рассматриваться (линейные) пространства $S_{p}^{w}(\mathbb{R}, \mathscr{M}(U))=S_{p}^{\left(\Phi_{0}\right)}(\mathbb{R}, \mathscr{M}(U)$ ) (слабо п.п. по Степанову мерозначных функций степени $p)$ и $\widetilde{S}_{p}^{w}(\mathbb{R}, \mathscr{M}(U))=S_{p}^{\left(\Phi_{a p}\right)}(\mathbb{R}, \mathscr{M}(U))\left(\right.$ будет показано, что $\widetilde{S}_{p}^{w}(\mathbb{R}, \mathscr{M}(U))=$ $S_{p}^{\left(\Phi_{\tau}\right)}(\mathbb{R}, \mathscr{M}(U))$ для всех $\left.\tau>0\right)$.

Для любых открытого множества $\mathscr{O} \subset U$ и мерозначной функции $\mu[\cdot ; \cdot] \in$ $S_{p}^{(\Phi)}(\mathbb{R}, \mathscr{M}(U))$ положим $\lambda_{\mu}^{(\Phi)}[\mathscr{O}]=\sup \left\{\|\mu(F(\cdot, \cdot) ; \cdot)\|_{M_{p}(\mathbb{R})}: F \in B_{1}(\Phi)\right.$ и при п.в. $t \in \mathbb{R} F(x, t)=0$ для всех $x \in U \backslash \mathscr{O}\}$.

ТЕорема 3. Пусть $(U, \rho)$ - полное сепарабельное метрическое пространство и $\mu[\cdot ; \cdot] \in S_{p}^{(\Phi)}(\mathbb{R}, \mathscr{M}(U)), p \geqslant 1, \Phi \in \mathscr{F}_{U}$. Тогда $\lambda_{\mu}^{(\Phi)}[U]<+\infty$ и для 
любого $\varepsilon>0$ существует компакт $K_{\varepsilon} \subset U$ такой, что $\lambda_{\mu}^{(\Phi)}\left[U \backslash K_{\varepsilon}\right]<\varepsilon$.

СЛЕДСТВИЕ. Пусть $(U, \rho)$ - полное сепарабельное метрическое пространство и либо $\mu[\cdot ; \cdot] \in S_{p}^{w}(\mathbb{R}, \mathscr{M}(U)) \quad$ и $\mu[\cdot ; t] \in \mathscr{M}^{+}(U)$ n.в., либо $\mu[\cdot ; \cdot] \in S_{p}^{\left(\Phi_{\tau}\right)}(\mathbb{R}, \mathscr{M}(U)), \tau>0, p \geqslant 1$. Тогда $|\mu|[U ; \cdot] \in M_{p}(\mathbb{R})$ и для любого $\varepsilon>0$ существует компакт $K_{\varepsilon} \subset U$ такой, что $\left\||\mu|\left[U \backslash K_{\varepsilon} ; \cdot\right]\right\|_{M_{p}(\mathbb{R})}<\varepsilon$.

Для доказательства следствия достаточно заметить, что для любого открытого множества $\mathscr{O} \subset U\|\mu[\mathscr{O} ; \cdot]\|_{M_{p}(\mathbb{R})}=\lambda_{\mu}^{\left(\Phi_{0}\right)}[\mathscr{O}]$, если $\mu[\cdot ; \cdot] \in S_{p}^{w}(\mathbb{R}, \mathscr{M}(U))$ и $\mu[\cdot ; t] \in \mathscr{M}^{+}(U)$ п.в., $\||\mu|[\mathscr{O} ; \cdot]\|_{M_{p}(\mathbb{R})} \leqslant(-[-1 / \tau])^{1 / p} \lambda_{\mu}^{\left(\Phi_{\tau}\right)}[\mathscr{O}]$, где $[\xi]-$ целая часть числа $\xi \in \mathbb{R}$, если $\mu[\cdot ; \cdot] \in S_{p}^{\left(\Phi_{\tau}\right)}(\mathbb{R}, \mathscr{M}(U)), \tau>0$.

Пусть $(\mathscr{H},\|\cdot\|)$ - банахово пространство и $T \subset \mathbb{R}-$ непустое измеримое множество. Обозначим через $\widetilde{Q} \mathscr{H}(T)$ множество последовательностей $\left\{f_{j}\right\}$ (определенных при п.в. $t \in T$ ) измеримых функций $f_{j}: T \rightarrow \mathscr{H}, j \in \mathbb{N}$, таких, что для любого непустого множества $J \subset \mathbb{N}$ ряд $\sum_{j \in J} f_{j}(t)=f(J ; t)$ сходится при п.в. $t \in T$.

Доказательство следующей теоремы, которая используется при доказательстве теоремы 3 , приведено в $\S 5$.

Tеорема 4. Пусть $(\mathscr{H},\|\cdot\|)$-банахово пространство, $\left\{f_{j}\right\} \in \widetilde{Q}_{\mathscr{H}}(\mathbb{R}), p \geqslant 1$. Предположим, что $f(J ; \cdot) \in S_{p}(\mathbb{R}, \mathscr{H})$ для любого непустого множества $J \subset \mathbb{N}$. Тогда

$$
\lim _{l \rightarrow+\infty} \sup _{J \in \mathscr{S}_{l}}\|f(J ; \cdot)\|_{M_{p}(\mathbb{R}, \mathscr{H})}=0
$$

ДоказАтЕльство теоремы 3. Так как линейное отображение $\Phi \ni F \rightarrow$ $\mu(F(\cdot, \cdot) ; \cdot) \in M_{p}(\mathbb{R})$ имеет замкнутый график, то оно непрерывно. Следовательно, $\lambda_{\mu}^{(\Phi)}[\mathscr{O}] \leqslant \lambda_{\mu}^{(\Phi)}[U]<+\infty$ для любого открытого множества $\mathscr{O} \subset U$ и полунорма $F \rightarrow \lambda_{\mu}^{(\Phi)}(F)=\sup _{G \in B_{1}(\Phi)}\|\mu(G(\cdot, \cdot) F(\cdot, \cdot) ; \cdot)\|_{M_{p}(\mathbb{R})}$ непрерьвна на банаховом пространстве $\left(\Phi,\|\cdot\|_{\infty}\right)$. Разобьем доказательство на несколько лемм.

Лемма 4. Пусть $F_{j} \in \Phi^{+}, j \in \mathbb{N}$, при п.в. $t \in \mathbb{R} F_{j}(x, t) \downarrow 0, j \rightarrow+\infty$, для всех $x \in U u\left\|F_{j}\left(\left.\cdot\right|_{K}, \cdot\right)\right\|_{M_{1}\left(\mathbb{R}, C_{b}(K)\right)} \rightarrow 0, j \rightarrow+\infty$, для любого непустого компакта $K \subset U$. Тогда

$$
\lim _{j \rightarrow+\infty}\left\|\mu\left(G(\cdot, \cdot) F_{j}(\cdot, \cdot) ; \cdot\right)\right\|_{M_{p}(\mathbb{R})}=0
$$

для любой функиии $G \in \Phi$.

ДоказАТЕЛЬСтво. Обозначим $f_{j}(t)=\mu\left(G(\cdot, t)\left(F_{j}(\cdot, t)-F_{j+1}(\cdot, t)\right) ; t\right), t \in \mathbb{R}$, $j \in \mathbb{N}$. Так как для любого непустого множества $J \subset \mathbb{N}$ функция $\mathbb{R} \ni t \rightarrow$ $\widetilde{F}_{J}(\cdot, t)=\sum_{j \in J} G(\cdot, t)\left(F_{j}(\cdot, t)-F_{j+1}(\cdot, t)\right) \in C_{b}(U)$ принадлежит пространству $L_{\infty}^{\circ}\left(\mathbb{R}, C_{b}(U)\right)$, ряд при п.в. $t \in \mathbb{R}$ сходится равномерно на компактах $K \subset U$ и $\left\|\widetilde{F}_{J}\left(\left.\cdot\right|_{K}, \cdot\right)-\widetilde{F}_{J \cap\{1, \ldots, l\}}\left(\left.\cdot\right|_{K}, \cdot\right)\right\|_{M_{1}\left(\mathbb{R}, C_{b}(K)\right)} \rightarrow 0$ при $l \rightarrow+\infty$ для любого непустого компакта $K \subset U$, то $\widetilde{F}_{J}(\cdot, \cdot) \in \Phi$ и, следовательно, $f(J ; \cdot) \in S_{p}(\mathbb{R})$, где $f(J ; t)=\sum_{j \in J} f_{j}(t), t \in \mathbb{R}$. С другой стороны,

$\left\|\mu\left(G(\cdot, \cdot) F_{j}(\cdot, \cdot) ; \cdot\right)\right\|_{M_{p}(\mathbb{R})}=\left\|\sum_{i \geqslant j} f_{i}(\cdot)\right\|_{M_{p}(\mathbb{R})} \leqslant \sup _{J \in \mathscr{S}_{j}}\|f(J ; \cdot)\|_{M_{p}(\mathbb{R})}, j \in \mathbb{N}$.

Поэтому равенство (3) следует из теоремы 4. Лемма доказана. 
Лемма 5. Пусть $F_{j} \in \Phi^{+}, j \in \mathbb{N}$, при п.в. $t \in \mathbb{R} F_{j}(x, t) \downarrow 0, j \rightarrow+\infty$, для всех $x \in U u\left\|F_{j}\left(\left.\cdot\right|_{K}, \cdot\right)\right\|_{M_{1}\left(\mathbb{R}, C_{b}(K)\right)} \rightarrow 0, j \rightarrow+\infty$, для любого непустого компакта $K \subset U$. Тогда $\lambda_{\mu}^{(\Phi)}\left(F_{j}\right) \rightarrow 0$ при $j \rightarrow+\infty$.

ДоказАТЕльство. Допустим противное. Тогда найдутся число $\varepsilon>0$ и подпоследовательность $\left\{F_{j_{k}}\right\}$ такие, что $\lambda_{\mu}^{(\Phi)}\left(F_{j_{k}}\right)>\varepsilon$ для всех $k \in \mathbb{N}$. Не ограничивая обшности, можно считать, что $\lambda_{\mu}^{(\Phi)}\left(F_{j}\right)>\varepsilon$ для самой последовательности $\left\{F_{j}\right\}$. Найдутся функции $G_{j} \in B_{1}(\Phi), j \in \mathbb{N}$, для которых $\left\|\mu\left(G_{j}(\cdot, \cdot) F_{j}(\cdot, \cdot) ; \cdot\right)\right\|_{M_{p}(\mathbb{R})}$ $>\varepsilon$. Определим последовательно при $s=1,2, \ldots$ числа $j_{s} \in \mathbb{N}$. Положим $j_{1}=1$. Если число $j_{s}$ при некотором $s \in \mathbb{N}$ уже определено, то число $j_{s+1}>j_{s}$ выберем в соответствии с леммой 4 так, что $\left\|\mu\left(G_{j_{s}}(\cdot, \cdot) F_{j_{s+1}}(\cdot, \cdot) ; \cdot\right)\right\|_{M_{p}(\mathbb{R})}<\varepsilon / 2$. Для любого непустого множества $J \subset \mathbb{N}$ при п.в. $t \in \mathbb{R}$ ряд $\sum_{s \in J} G_{j_{s}}(x, t)\left(F_{j_{s}}(x, t)-\right.$ $\left.F_{j_{s+1}}(x, t)\right), x \in U$, сходится равномерно на компактных множествах $K \subset U$ к некоторой функции $(x, t) \rightarrow \widetilde{F}_{J}(x, t)$ из пространства $L_{\infty}^{\circ}\left(\mathbb{R}, C_{b}(U)\right)$, при этом $\left\|\widetilde{F}_{J}\left(\left.\cdot\right|_{K}, \cdot\right)-\widetilde{F}_{J \cap\{1, \ldots, l\}}\left(\left.\cdot\right|_{K}, \cdot\right)\right\|_{M_{1}\left(\mathbb{R}, C_{b}(K)\right)} \rightarrow 0$ при $l \rightarrow+\infty$ для любого непустого компакта $K \subset U$. Поэтому $\widetilde{F}_{J}(\cdot, \cdot) \in \Phi$ и $\mu\left(\widetilde{F}_{J}(\cdot, \cdot) ; \cdot\right) \in S_{p}(\mathbb{R})$. Из теоремы 4 получаем, что $\left\|\mu\left(\widetilde{F}_{\{s\}}(\cdot, \cdot) ; \cdot\right)\right\|_{M_{p}(\mathbb{R})} \rightarrow 0$ при $s \rightarrow+\infty$. С другой стороны, для всех $s \in \mathbb{N}$

$$
\begin{aligned}
\left\|\mu\left(\widetilde{F}_{\{s\}}(\cdot, \cdot) ; \cdot\right)\right\|_{M_{p}(\mathbb{R})} \geqslant & \left\|\mu\left(G_{j_{s}}(\cdot, \cdot) F_{j_{s}}(\cdot, \cdot) ; \cdot\right)\right\|_{M_{p}(\mathbb{R})} \\
& -\left\|\mu\left(G_{j_{s}}(\cdot, \cdot) F_{j_{s+1}}(\cdot, \cdot) ; \cdot\right)\right\|_{M_{p}(\mathbb{R})} \\
> & \varepsilon-\frac{\varepsilon}{2}=\frac{\varepsilon}{2} .
\end{aligned}
$$

Полученное противоречие доказывает лемму.

Отметим, что для любого открытого множества $\mathscr{O} \subset U \lambda_{\mu}^{(\Phi)}[\mathscr{O}]=\sup \left\{\lambda_{\mu}^{(\Phi)}(F)\right.$ : $F \in B_{1}(\Phi)$ и при п.в. $t \in \mathbb{R} F(x, t)=0$ для всех $\left.x \in U \backslash \mathscr{O}\right\}$. Кроме того, если $G \in \Phi$ и при п.в. $t \in \mathbb{R} G(x, t)=1$ для всех $x \in \mathscr{O}$, то $\lambda_{\mu}^{(\Phi)}[\mathscr{O}] \leqslant \lambda_{\mu}^{(\Phi)}(G)$.

Лемма 6. Для любых открытых множеств $\mathscr{O}_{i} \subset U, i \in \mathbb{N}$, справедливо неравенство

$$
\lambda_{\mu}^{(\Phi)}\left[\bigcup_{i=1}^{+\infty} \mathscr{O}_{i}\right] \leqslant \sum_{i=1}^{+\infty} \lambda_{\mu}^{(\Phi)}\left[\mathscr{O}_{i}\right]
$$

ДокАЗАТЕльство. Покажем вначале, что

$$
\lambda_{\mu}^{(\Phi)}\left[\bigcup_{i \leqslant j} \mathscr{O}_{i}\right] \leqslant \sum_{i \leqslant j} \lambda_{\mu}^{(\Phi)}\left[\mathscr{O}_{i}\right], \quad j \in \mathbb{N}
$$

Выберем функцию $F \in B_{1}(\Phi)$ такую, что при п.в. $t \in \mathbb{R} \quad F(x, t)=0$ для всех $x \in U \backslash \bigcup_{i \leqslant j} \mathscr{O}_{i}$. Определим функции $\Theta_{i}(x)=\rho\left(x, U \backslash \mathscr{O}_{i}\right)\left(\sum_{k \leqslant j} \rho\left(x, U \backslash \mathscr{O}_{k}\right)\right)^{-1}$, если $x \in \bigcup_{i \leqslant j} \mathscr{O}_{i}$, и $\Theta_{i}(x)=0$, если $x \in U \backslash \bigcup_{i \leqslant j} \mathscr{O}_{i}, i=1, \ldots, j$. Имеем $\Theta_{i}(\cdot) F(\cdot, \cdot) \in \Phi$ и при п.в. $t \in \mathbb{R} \Theta_{i}(x) F(x, t)=0$ для всех $x \notin \mathscr{O}_{i}, i=1, \ldots, j$, $F(x, t)=\sum_{i \leqslant j} \Theta_{i}(x) F(x, t), x \in U$. Отсюда

$$
\lambda_{\mu}^{(\Phi)}(F) \leqslant \sum_{i \leqslant j} \lambda_{\mu}^{(\Phi)}\left(\Theta_{i} F\right) \leqslant \sum_{i \leqslant j} \lambda_{\mu}^{(\Phi)}\left[\mathscr{O}_{i}\right]
$$


Из произвольности выбора функции $F$ следует неравенство (5). Обозначим $Y_{j}(x)=\max \left\{0,1-j \rho\left(x, U \backslash \bigcup_{i \leqslant j} \mathscr{O}_{i}\right)\right\}, x \in U, j \in \mathbb{N} ; Y_{j} \in C_{b}^{+}(U)$ и $Y_{j}(x) \downarrow 0$, $j \rightarrow+\infty$, для всех $x \in \bigcup_{i \in \mathbb{N}} \mathscr{O}_{i}$. Выберем любую функцию $F \in B_{1}(\Phi)$, для которой при п.в. $t \in \mathbb{R} F(x, t)=0$ для всех $x \notin \bigcup_{i \in \mathbb{N}} \mathscr{O}_{i}$. Тогда $F^{ \pm}= \pm \frac{1}{2}(F \pm|F|) \in \Phi$, при п.в. $t \in \mathbb{R} \quad Y_{j}(\cdot) F^{ \pm}(\cdot, t) \in C_{b}^{+}(U)$ и $Y_{j}(x) F^{ \pm}(x, t) \downarrow 0, j \rightarrow+\infty$, для всех $x \in U$. Из свойства 4$)$, характеризующего подпространство $\Phi$, следует, что $\left\|Y_{j}\left(\left.\cdot\right|_{K}\right) F^{ \pm}\left(\left.\cdot\right|_{K}, \cdot\right)\right\|_{M_{1}\left(\mathbb{R}, C_{b}(K)\right)} \rightarrow 0$ при $j \rightarrow+\infty$ для любого непустого компакта $K \subset U$. Поэтому (в силу леммы 5 )

$$
0 \leqslant \lambda_{\mu}^{(\Phi)}\left(Y_{j} F\right) \leqslant \lambda_{\mu}^{(\Phi)}\left(Y_{j} F^{+}\right)+\lambda_{\mu}^{(\Phi)}\left(Y_{j} F^{-}\right) \rightarrow 0
$$

при $j \rightarrow+\infty$. Так как

$$
\lambda_{\mu}^{(\Phi)}\left(\left(1-Y_{j}\right) F\right) \leqslant \lambda_{\mu}^{(\Phi)}\left[\bigcup_{i \leqslant j} \mathscr{O}_{i}\right] \leqslant \sum_{i \leqslant j} \lambda_{\mu}^{(\Phi)}\left[\mathscr{O}_{i}\right] \leqslant \sum_{i \in \mathbb{N}} \lambda_{\mu}^{(\Phi)}\left[\mathscr{O}_{i}\right], \quad j \in \mathbb{N},
$$

то из $(6)$ и $(7)$ при $j \rightarrow+\infty$ получаем $\lambda_{\mu}^{(\Phi)}(F) \leqslant \sum_{i=1}^{+\infty} \lambda_{\mu}^{(\Phi)}\left[\mathscr{O}_{i}\right]$. Неравенство (4) следует из полученной оценки и произвольности выбора функции $F$. Лемма доказана.

Пусть точки $x_{j} \in U, j \in \mathbb{N}$, образуют счетное плотное множество в пространстве $U$. Для всех $j, n \in \mathbb{N}$ определим функции $U \ni x \rightarrow \Theta_{j}^{n}(x) \in \mathbb{R}$ : $\Theta_{j}^{n}(x)=0$, если $\rho\left(x, \bigcup_{i \leqslant j} x_{i}\right) \leqslant 1 /(2 n), \Theta_{j}^{n}(x)=2 n \rho\left(x, \bigcup_{i \leqslant j} x_{i}\right)-1$, если $1 /(2 n)<\rho\left(x, \bigcup_{i \leqslant j} x_{i}\right) \leqslant 1 / n$, и $\Theta_{j}^{n}(x)=1$, если $\rho\left(x, \bigcup_{i \leqslant j} x_{i}\right)>1 / n$. Имеем $\Theta_{j}^{n} \in C_{b}^{+}(U)$ и $\Theta_{j}^{n}(x) \downarrow 0$ при $j \rightarrow+\infty$ для всех $x \in U$ (и $n \in \mathbb{N}$ ). Обозначим $\Theta_{j}^{n}(x, t)=\Theta_{j}^{n}(x), x \in U, t \in \mathbb{R}$. Выберем любое $\varepsilon>0$. Из леммы 5 следует, что для любого $n \in \mathbb{N}$ можно найти такое число $j(n) \in \mathbb{N}$, что $\lambda_{\mu}^{(\Phi)}\left(\Theta_{j(n)}^{n}(\cdot, \cdot)\right)<$ $2^{-n} \varepsilon$. Обозначим $\widetilde{K}_{n}=\left\{x \in U: \rho\left(x, \bigcup_{i \leqslant j(n)} x_{i}\right) \leqslant 1 / n\right\}, K_{\varepsilon}=\bigcap_{n \in \mathbb{N}} \widetilde{K}_{n}$. Множество $K_{\varepsilon}$ компактное. Так как $\lambda_{\mu}^{(\Phi)}\left[U \backslash \widetilde{K}_{n}\right] \leqslant \lambda_{\mu}^{(\Phi)}\left(\Theta_{j(n)}^{n}(\cdot, \cdot)\right)<2^{-n} \varepsilon$ для всех $n \in \mathbb{N}$, то из леммы 6 получаем

$$
\lambda_{\mu}^{(\Phi)}\left[U \backslash K_{\varepsilon}\right] \leqslant \sum_{n=1}^{+\infty} \lambda_{\mu}^{(\Phi)}\left[U \backslash \widetilde{K}_{n}\right]<\sum_{n=1}^{+\infty} 2^{-n} \varepsilon=\varepsilon
$$

Теорема 3 доказана.

ТеОРема 5. Пусть $(U, \rho)$ - полное сепарабельное метрическое пространство $и \mathbb{R} \ni t \rightarrow \mu[\cdot ; t] \in \mathscr{M}(U)-$ измеримая мерозначная функция. Предположим, что $|\mu|[U ; \cdot] \in M_{p}(\mathbb{R})$ и для любого $\varepsilon>0$ существует компакт $K_{\varepsilon} \subset U$ такой, что $\left\||\mu|\left[U \backslash K_{\varepsilon} ; \cdot\right]\right\|_{M_{p}(\mathbb{R})}<\varepsilon, p \geqslant 1$. Тогда следующие условия эквивалентны:

1) $\mu[\cdot ; \cdot] \in S_{p}^{w}(\mathbb{R}, \mathscr{M}(U))$;

2) $\mu[\cdot ; \cdot] \in S_{p}\left(\mathbb{R},\left(\mathscr{M}(U),\|\cdot\|_{w}\right)\right)$.

При выполнении этих условий

$$
\operatorname{Mod} \mu[\cdot ; \cdot]=\sum_{F \in \mathscr{L}(U)} \operatorname{Mod} \mu(F ; \cdot)=\sum_{F \in C_{b}(U)} \operatorname{Mod} \mu(F ; \cdot) .
$$


ДокАЗАТЕЛЬСтво. 2$) \Rightarrow 1$ ). Если $G \in \mathscr{L}(U)$, то всякий $\varepsilon$-почти период функции $\mu[\cdot ; \cdot] \in S_{p}(\mathbb{R}, \mathscr{M}(U))$ является также $\varepsilon$-почти периодом функции $\mu(G ; \cdot) \in M_{p}(\mathbb{R})$. Поэтому $\mu(G ; \cdot) \in S_{p}(\mathbb{R})$ и $\operatorname{Mod} \mu(G ; \cdot) \subset \operatorname{Mod} \mu[\cdot ; \cdot]$. Пусть $F \in C_{b}(U)$. Для любого $\varepsilon>0$ сушествует функция $G_{\varepsilon} \in C_{b}(U)$ такая, что $\lambda G_{\varepsilon}+\gamma \in \mathscr{L}(U)$ для некоторых $\lambda>0$ и $\gamma \in \mathbb{R},\left\|G_{\varepsilon}\right\|_{C_{b}(U)} \leqslant\|F\|_{C_{b}(U)}$ и $\max _{x \in K_{\varepsilon}}\left|F(x)-G_{\varepsilon}(x)\right|<\varepsilon$. Таккак $\mu\left(G_{\varepsilon} ; \cdot\right) \in S_{p}(\mathbb{R}), \operatorname{Mod} \mu\left(G_{\varepsilon} ; \cdot\right) \subset \operatorname{Mod} \mu[\cdot ; \cdot],\left\|\mu(F ; \cdot)-\mu\left(G_{\varepsilon} ; \cdot\right)\right\|_{M_{p}(\mathbb{R})} \leqslant$ $\left(2\|F\|_{C_{b}(U)}+\||\mu|[U ;,]\|_{M_{p}(\mathbb{R})}\right) \varepsilon$ и число $\varepsilon>0$ можно выбирать сколь угодно малым, то $\mu(F ; \cdot) \in S_{p}(\mathbb{R})$ и $\operatorname{Mod} \mu(F ; \cdot) \subset \sum_{G \in \mathscr{L}(U)} \operatorname{Mod} \mu(G ; \cdot) \subset \operatorname{Mod} \mu[\cdot ; \cdot]$.

$1) \Rightarrow 2)$. Воспользуемся схемой доказательства импликации 1) $\Rightarrow 2)$ в теореме 1. Так как $\|\mu[\cdot ; t]\|_{w} \leqslant|\mu|[U ; t]$ п.в., то $\mu[\cdot ; \cdot] \in M_{p}\left(\mathbb{R},\left(\mathscr{M}(U),\|\cdot\|_{w}\right)\right)$. Пусть $\varepsilon>0$. Множество $\mathscr{L}\left(K_{\varepsilon}\right)$ компактное, поэтому найдутся функции $G_{j}^{(\varepsilon)} \in \mathscr{L}(U)$, $j=1, \ldots, n(\varepsilon)$, для которых функции $G_{j}^{(\varepsilon)}\left(\left.\cdot\right|_{K_{\varepsilon}}\right)$ образуют $\varepsilon$-сеть для $\mathscr{L}\left(K_{\varepsilon}\right)$. Пусть $\tau$ - общий $\frac{\varepsilon}{n(\varepsilon)}$-почти период функций $\mu\left(G_{j}^{(\varepsilon)} ; \cdot\right) \in S_{p}(\mathbb{R}), j=1, \ldots, n(\varepsilon)$ (множество таких чисел относительно плотно). При п.в. $t \in \mathbb{R}$ имеем

$$
\begin{aligned}
& \sup _{G \in \mathscr{L}(U)}|\mu(G ; \cdot)-\mu(G ; t+\tau)| \\
& \leqslant \varepsilon|\mu|[U ; t]+\varepsilon|\mu|[U ; t+\tau]+|\mu|\left[U \backslash K_{\varepsilon} ; t\right]+|\mu|\left[U \backslash K_{\varepsilon} ; t+\tau\right] \\
& \quad+\max _{j=1, \ldots, n(\varepsilon)}\left|\mu\left(G_{j}^{(\varepsilon)} ; t\right)-\mu\left(G_{j}^{(\varepsilon)} ; t+\tau\right)\right|,
\end{aligned}
$$

поэтому

$$
\begin{aligned}
& \|\mu[\cdot ; \cdot]-\mu[\cdot ; \cdot+\tau]\|_{M_{p}(\mathbb{R}, \mathscr{M}(U))} \\
& \quad \leqslant 2 \varepsilon+2 \varepsilon\||\mu|[U ; \cdot]\|_{M_{p}(\mathbb{R})}+\sum_{j=1}^{n(\varepsilon)}\left\|\mu\left(G_{j}^{(\varepsilon)} ; \cdot\right)-\mu\left(G_{j}^{(\varepsilon)} ; \cdot+\tau\right)\right\|_{M_{p}(\mathbb{R})} \\
& \quad<3 \varepsilon+2 \varepsilon\||\mu|[U ; \cdot]\|_{M_{p}(\mathbb{R})} .
\end{aligned}
$$

В силу произвольности выбора числа $\varepsilon>0$ отсюда следует $\mu[\cdot ; \cdot] \in S_{p}(\mathbb{R}, \mathscr{M}(U))$. Если $\varepsilon_{i} \rightarrow 0$ при $i \rightarrow+\infty$, то всякая $\mu\left(G_{j}^{\left(\varepsilon_{i}\right)} ; \cdot\right)$-возвращающая для всех $j=1, \ldots, n\left(\varepsilon_{i}\right), i \in \mathbb{N}$, последовательность $\left\{\tau_{l}\right\}$ является также $\mu[\cdot ; \cdot]$-возвращающей, поэтому $\operatorname{Mod} \mu[\cdot ; \cdot] \subset \sum_{G \in \mathscr{L}(U)} \operatorname{Mod} \mu(G ; \cdot) \subset \sum_{F \in C_{b}(U)} \operatorname{Mod} \mu(F ; \cdot)$. Теорема доказана.

СЛЕДСТВИЕ. Пусть $(U, \rho)$ - полное сепарабельное метрическое пространство, $\mathbb{R} \ni t \rightarrow \mu[\cdot ; t] \in \mathscr{M}^{+}(U)-$ произвольная мерозначная функиия, $p \geqslant 1$. Тогда следующие условия әквивалентны:

1) $\mu[\cdot ; \cdot] \in S_{p}^{w}(\mathbb{R}, \mathscr{M}(U))$;

2) $\mu[\cdot ; \cdot] \in S_{p}\left(\mathbb{R},\left(\mathscr{M}(U),\|\cdot\|_{w}\right)\right)$.

ДокаЗАТЕльство. Импликация 1) $\Rightarrow 2$ ) вытекает из теоремы 5 и следствия теоремы 3. Импликация 2) $\Rightarrow 1$ ) непосредственно следует из леммы 2 (так как пространство $\left(\mathscr{M}^{+}(U),\|\cdot\|_{w}\right)$ полное и для любой функции $F \in C_{b}(U)$ функция $\mathscr{M}^{+}(U) \ni \mu \rightarrow \widetilde{F}(\mu)=\mu(F) \in \mathbb{R}$ непрерывна и $|\widetilde{F}(\mu)| \leqslant\|F\|_{C_{b}(U)}\|\mu\|_{w}$, $\left.\mu \in \mathscr{M}^{+}(U)\right)$. 
ЗАмЕчАНИЕ. Из следствия теоремы 5 и результатов работы [14] (а также из [7; теорема 1]) вытекает следующее утверждение: если $(U, \rho)$ - полное сепарабельное метрическое пространство, $\mu[\cdot ; \cdot] \in S_{p}(\mathbb{R}, \mathscr{M}(U)), p \geqslant 1$, и $\mu[\cdot ; t] \in \mathscr{M}^{+}(U)$ п.в., то для любого $\varepsilon>0$ сушествуют измеримое множество $T \subset \mathbb{R}$ и множество $A \in \mathscr{K}_{U}$ такие, что $\chi_{T} \in S_{1}(\mathbb{R})\left(\chi_{T}-\right.$ характеристическая функция множества $\left.T\right)$, $\operatorname{Mod} \chi_{T} \subset \operatorname{Mod} \mu[\cdot ; \cdot], \sup _{t \in \mathbb{R}} m([t, t+1] \backslash T)<\varepsilon, \mu[\cdot ; t] \in A$ для всех $t \in T$ и $\left\|\chi_{\mathbb{R} \backslash T}(\cdot) \mu[U ; \cdot]\right\|_{M_{p}(\mathbb{R})}<\varepsilon$.

Приведем примеры, показывающие, что условия 1) и 2) из теоремы 5 могут независимо выполняться или не выполняться, если условия теоремы 5 не выполнены.

В примере 1 приведены компактное метрическое пространство $(U, \rho)$ и измеримая мерозначная функция $[0,1) \ni t \rightarrow \widetilde{\mu}[\cdot ; t] \in \mathscr{M}(U)$ такие, что $|\widetilde{\mu}|[U ; \cdot] \notin L_{p}([0,1), \mathbb{R}), \tilde{\mu}[\cdot ; \cdot] \notin L_{p}([0,1), \mathscr{M}(U))$, но $\widetilde{\mu}(F ; \cdot) \in L_{p}([0,1), \mathbb{R})$ для всех $F \in C_{b}(U)$. Если $t \rightarrow \mu[\cdot ; t]$ - периодическое с единичным периодом продолжение на $\mathbb{R}$ функции $\widetilde{\mu}[\cdot ; \cdot]$, то $|\mu|[U ; \cdot] \notin M_{p}(\mathbb{R}), \mu[\cdot ; \cdot] \notin S_{p}(\mathbb{R}, \mathscr{M}(U))$, но $\mu[\cdot ; \cdot] \in S_{p}^{w}(\mathbb{R}, \mathscr{M}(U))$.

Пример 1. Пусть $(U, \rho)$ - замкнутьй единичньй шар в $\mathbb{R}^{d}, d \geqslant 4 p, p \geqslant 1$, с евклидовой метрикой $\rho$. Для $\varepsilon \in(0,1]$ обозначим через $k(\varepsilon)$ максимальное число точек $x_{1}(\varepsilon), \ldots, x_{k(\varepsilon)}(\varepsilon) \in U$ таких, что $\rho\left(x_{i_{1}}(\varepsilon), x_{i_{2}}(\varepsilon)\right) \geqslant \varepsilon$ для всех $i_{1} \neq i_{2}$. Справедлива оценка $k(\varepsilon) \geqslant \varepsilon^{-d}$. Определим функции $\mathbb{R} \ni t \rightarrow f_{j}(t) \in \mathbb{R}, j \in \mathbb{N}$ : если $t \notin[0,1)$, то положим $f_{j}(t)=0$, если $t \in[0,1)$, то $f_{j}(t)=-1$ в случае $t \in\left[(s-1) 2^{-j}, s 2^{-j}\right)$, где $s \in 2 \mathbb{N}-1$ и и $f_{j}(t)=1$ в случае $t \in\left[(s-1) 2^{-j}, s 2^{-j}\right)$, $s \in 2 \mathbb{N}$. Обозначим $n_{l}=\sum_{s=0}^{l}(2 s)^{4}, l \in \mathbb{Z}_{+}$. Для всех $n \in \mathbb{N}$ на полуинтервале $[0,1)$ определим функции $\widetilde{f}_{n}$ : если $n_{l-1}<n \leqslant n_{l}, l=l(n) \in \mathbb{N}$, то положим $\widetilde{f}_{n}(t)=2^{l / p}(2 l)^{-4} f_{n_{l}-n+1}\left(2^{l} t-1\right), t \in[0,1)$. Так как для всех $a_{j} \in[-1,1]$, $j=1, \ldots, 2 m, m \in \mathbb{N}$, имеем

$$
\begin{aligned}
\left\|\sum_{j=1}^{2 m} a_{j} f_{j}\right\|_{L_{p}([0,1), \mathbb{R})}^{p} & =2^{-2 m} \sum_{ \pm}\left| \pm a_{1} \pm \cdots \pm a_{2 m}\right|^{p} \\
& \leqslant 2^{-2 m} \sum_{ \pm}| \pm 1 \pm \cdots \pm 1|^{p} \leqslant c_{p}(2 m)^{p / 2}
\end{aligned}
$$

(суммирование производится по всем комбинациям знаков), где константа $c_{p}>0$ зависит от $p$, то для всех $a_{n} \in[-1,1], n \in \mathbb{N}$, справедлива оценка

$$
\left\|\sum_{n=1}^{+\infty} a_{n} \widetilde{f}_{n}\right\|_{L_{p}([0,1), \mathbb{R})}^{p} \leqslant c_{p} \sum_{n=1}^{+\infty}(2 l)^{-2 p}<+\infty .
$$

Пусть $\varepsilon_{l}=(2 l)^{-4 / d}, l \in \mathbb{N}$. Обозначим $j(n)=n_{l(n)}-n+1, j(n) \in\left\{1, \ldots,(2 l(n))^{4}\right\}$. Положим $\widetilde{\mu}[\cdot ; t]=\sum_{n=1}^{+\infty} \tilde{f}_{n}(t) \delta_{x_{j(n)}\left(\varepsilon_{l(n)}\right)}[\cdot], t \in[0,1)$ (где $\delta_{x}[\cdot]-$ мера Дирака, сосредоточенная в точке $x \in U)$. Из (8) следует, что $\widetilde{\mu}(F ; \cdot) \in L_{p}([0,1), \mathbb{R})$ для всех функций $F \in C_{b}(U)$. С другой стороны, для всех $t \in\left[2^{-l}, 2^{-l+1}\right), l \in \mathbb{N}$, справедлива оценка $\|\widetilde{\mu}[\cdot ; t]\|_{w} \geqslant \frac{1}{2} \varepsilon_{l}|\widetilde{\mu}|[U ; t]-\frac{1}{2} \varepsilon_{l} \widetilde{\mu}[U ; t]$, при этом $|\widetilde{\mu}|[U ; t]=2^{l / p}$. Следовательно, $\||\widetilde{\mu}|[U ; \cdot]\|_{L_{p}([0,1), \mathbb{R})}=+\infty$ и

$$
\|\widetilde{\mu}[\cdot ; \cdot]\|_{L_{p}([0,1), \mathscr{M}(U))} \geqslant \frac{1}{2}\left(\sum_{l=1}^{+\infty} \varepsilon_{l}^{p}\right)^{1 / p}-\frac{1}{2}\left(\sum_{l=1}^{+\infty} \varepsilon_{l}^{p}(2 l)^{-2 p}\right)^{1 / p}=+\infty .
$$


ПримеР 2. Пусть $(U, \rho)=\mathbb{N}$ с метрикой $\rho\left(n_{1}, n_{2}\right)=2$ при всех $n_{1} \neq n_{2}$ (в этом случае $\|\mu\|_{w}=|\mu|[U]$ для всех мер $\left.\mu \in \mathscr{M}(U)\right)$. Положим $a_{l}=\frac{1}{3}\left((-2)^{l-1}-1\right), l \in \mathbb{N}$. Множества $A_{l}=a_{l}+2^{l} \mathbb{Z}$ при разных $l \in \mathbb{N}$ не пересекаются и $\bigcup_{l \in \mathbb{N}} A_{l}=\mathbb{Z}[18]$. Пусть $[0,1) \ni t \rightarrow \widetilde{f}_{n}(t) \in \mathbb{R}, n \in \mathbb{N},-$ функции из примера 1. Для всех $t \in \mathscr{A}_{l}=$ $A_{l}+[0,1), l \in \mathbb{N}$, положим $\mu[\cdot ; t]=\sum_{n=n_{l-1}+1}^{n_{l}} \widetilde{f}_{n}(t-[t]) \delta_{n}[\cdot]([t]$ - целая часть числа $t)$. Так как $\int_{m}^{m+1}(|\mu|[U ; t])^{p} d t=1$ для всех $m \in \mathbb{Z}$, то $|\mu|[U ; \cdot] \in M_{p}(\mathbb{R})$. Пусть $F \in C_{b}(U)$ и $a_{n}=F(n), n \in \mathbb{N}$. Тогда $\mu(F ; t)=\sum_{n=n_{l-1}+1}^{n_{l}} a_{n} \widetilde{f}_{n}(t-[t])$ при $t \in \mathscr{A}_{l}, l \in \mathbb{N}$. Положим $g_{s}(t)=\mu(F ; t)$, если $t \in \bigcup_{l \leqslant s} \mathscr{A}_{l}$, и $g_{s}(t)=0$ в противном случае, $s \in \mathbb{N}$. Функция $g_{s}(\cdot)$ - периодическая с периодом $2^{s}$. Из неравенства

$$
\left\|\sum_{n=n_{l-1}+1}^{n_{l}} a_{n} \tilde{f}_{n}\right\|_{L_{p}([0,1), \mathbb{R})}^{p} \leqslant c_{p}(2 l)^{-2 p}\|F\|_{C_{b}(U)}
$$

получаем $\left\|\mu(F ; \cdot)-g_{s}(\cdot)\right\|_{M_{p}(\mathbb{R})} \rightarrow 0$ при $s \rightarrow+\infty$. Поэтому $\mu(F ; \cdot) \in S_{p}(\mathbb{R})$ для всех функций $F \in C_{b}(U)$. С другой стороны,

$$
\left\|\mu\left[\cdot ; \cdot+a_{m}\right]-\mu\left[\cdot ; \cdot+a_{l}\right]\right\|_{M_{p}(\mathbb{R}, \mathscr{M}(U))} \geqslant 2^{1 / p}
$$

для всех $m, l \in \mathbb{N}, m \neq l$, поэтому $\mu[\cdot ; \cdot] \notin S_{p}(\mathbb{R}, \mathscr{M}(U))$.

ПримеР 3. Пусть $U=[0,1], \rho(x, y)=|x-y|, x, y \in[0,1], p \geqslant 1$. Рассмотрим мерозначную функцию $\mathbb{R} \ni t \rightarrow \mu[\cdot ; t]=\sum_{l=1}^{+\infty} l^{3 / 4} \chi_{\mathscr{A}_{l}}(t)\left(\delta_{1 / l}[\cdot]-\delta_{0}[\cdot]\right)$, где $\chi \mathscr{A}_{l}$ - характеристические функции множеств $\mathscr{A}_{l}$, определенных в примере 2. Имеем $|\mu|[U ; \cdot] \notin M_{p}(\mathbb{R})$. Пусть $\mu_{s}[\cdot ; t]=\mu[\cdot ; t]$, если $t \in \bigcup_{l \leqslant s} \mathscr{A}_{l}$, и $\mu_{s}[\cdot ; t]=0$ в противном случае, $s \in \mathbb{N}$. Так как функции $t \rightarrow \mu_{s}[\cdot ; t]-$ периодические (с периодом $\left.2^{s}\right)$ и $\|\mu[\cdot ; t]\|_{w}=l^{-1 / 4}$ для всех $t \in \mathscr{A}_{l}$, то $\left\|\mu[\cdot ; \cdot]-\mu_{s}[\cdot ; \cdot]\right\|_{M_{p}(\mathbb{R}, \mathscr{M}(U))} \rightarrow 0$ при $s \rightarrow+\infty$. Следовательно, $\mu[\cdot ; \cdot] \in S_{p}(\mathbb{R}, \mathscr{M}(U))$. Выберем функцию $F(x)=\sqrt{x}, x \in[0,1]$. Тогда $\mu(F ; t)=l^{1 / 4}$ для всех $t \in \mathscr{A}_{l}, l \in \mathbb{N}$, поэтому $\mu(F ; \cdot) \notin M_{p}(\mathbb{R})$.

Пример 4. Пусть $U=\left\{x_{l}, y_{l}\right\}_{l \in \mathbb{N}}$. Обозначим $\mathscr{C}_{l}=\left\{x_{l}, y_{l}\right\}, l \in \mathbb{N}$. Положим $\rho\left(x_{l}, y_{l}\right)=1 / l$; если точки $z_{1}, z_{2} \in U$ принадлежат разньп множествам $\mathscr{C}_{l}$, то полагаем $\rho\left(z_{1}, z_{2}\right)=1$. Рассмотрим мерозначную функцию $\mathbb{R} \ni t \rightarrow \mu[\cdot ; \cdot]=$ $\sum_{l \in \mathbb{N}} \chi_{\mathscr{A}_{l}}(t)\left(\delta_{x_{l}}[\cdot]-\delta_{y_{l}}[\cdot]\right) \in \mathscr{M}(U)$. Так как $|\mu|[U ; t]=2$ и $\|\mu[\cdot ; t]\|_{w}=1 / l$ для всех $t \in \mathscr{A}_{l}, l \in \mathbb{N}$, то $|\mu|[U ; \cdot] \in M_{p}(\mathbb{R})$ и (см. пример 3) $\mu[\cdot ; \cdot] \in S_{p}(\mathbb{R}, \mathscr{M}(U))$, $p \geqslant 1$. Выберем функцию $F \in C_{b}(U)$ так, что $F\left(x_{l}\right)=(-1)^{l}$ и $F\left(y_{l}\right)=0$ для всех $l \in \mathbb{N}$. Тогда $\mu(F ; t)=(-1)^{l}$, если $t \in \mathscr{A}_{l}, l \in \mathbb{N}$. Следовательно (см. [18; пример 2.1]), $\mu(F ; \cdot) \notin S_{p}(\mathbb{R})$.

Для мерозначных функций $\mu[\cdot ; \cdot]$ из примеров 2 и $4|\mu|[U ; \cdot] \in M_{p}(\mathbb{R}), p \geqslant 1$, но не сушествует компакта $K \subset U$ такого, что $\||\mu|[U \backslash K ; \cdot]\|_{M_{p}(\mathbb{R})} \leqslant 1$.

ЛЕмма 7. Пусть $(U, \rho)$ - полное сепарабельное метрическое пространство, $\mathbb{R} \ni t \rightarrow \mu[\cdot ; t] \in \mathscr{M}(U)-($ п.в. определенная) мерозначная функиия, $p \geqslant 1, \tau>0$. Предположим, что $\mu(F(\cdot, \cdot) ; \cdot) \in M_{p}^{\prime}(\mathbb{R})$ для всех функиий $F(\cdot, \cdot) \in \Phi_{\tau}$. Тогда $|\mu|[U ; \cdot] \in M_{p}^{\prime}(\mathbb{R})$. 
ДоКАЗАТЕЛЬСТво. Не ограничивая общности, можно считать, что $\tau=1$. Линейное отображение $\left(\Phi_{1},\|\cdot\|_{\infty}\right) \ni F(\cdot, \cdot) \rightarrow \mu(F(\cdot, \cdot) ; \cdot) \in M_{p}(\mathbb{R})$ имеет замкнутый график и, следовательно, непрерывно. Так как для любых $\xi \in \mathbb{R}$ и $\varepsilon>0$ существует функция $F(\cdot, \cdot) \in B_{1}\left(\Phi_{1}\right)$ (можно считать, что $t \rightarrow F(\cdot, t) \in$ $\left(C_{b}(U),\|\cdot\|_{C_{b}(U)}\right)$ - элементарная функция) такая, что $\mu(F(\cdot, t) ; t) \geqslant|\mu|[U ; t]-\varepsilon$ при п.в. $t \in[\xi, \xi+1]$, то $|\mu|[U ; \cdot] \in M_{p}(\mathbb{R})$. Допустим, что доказываемое утверждение неверно. Тогда найдется число $\delta>0$ такое, что для любого $\varepsilon>0$ существуют число $n_{\varepsilon} \in \mathbb{Z}$, измеримое множество $T_{\varepsilon}\left(n_{\varepsilon}\right) \subset\left[n_{\varepsilon}, n_{\varepsilon}+1\right)$ и функция $F_{\varepsilon}(\cdot, \cdot) \in B_{1}\left(\Phi_{1}\right)$ такие, что $m\left(T_{\varepsilon}\left(n_{\varepsilon}\right)\right) \leqslant \varepsilon$ и $\int_{T_{\varepsilon}\left(n_{\varepsilon}\right)}\left|\mu\left(F_{\varepsilon}(\cdot, t) ; t\right)\right|^{p} d t \geqslant \delta$. Обозначим $T_{\varepsilon}(n)=T_{\varepsilon}\left(n_{\varepsilon}\right)+n-n_{\varepsilon}, n \in \mathbb{Z}, T_{\varepsilon}=T_{\varepsilon}\left(n_{\varepsilon}\right)+n \mathbb{Z}$. Положим $\varepsilon(1)=1$. Определим последовательно при $j=2,3, \ldots$ числа $\varepsilon(j) \in(0,1 / j]$ так, что

$$
\sup _{n \in \mathbb{Z}} \int_{T_{\varepsilon(j)}(n)}\left|\mu\left(F_{\varepsilon(s)}(\cdot, t) ; t\right)\right|^{p} d t<2^{-1-j+s} \delta, \quad s=1, \ldots, j-1 .
$$

Обозначим $\widetilde{T}_{j}=T_{\varepsilon(j)} \backslash \bigcup_{s \geqslant j+1} T_{\varepsilon(s)}, \widetilde{T}_{j}(n)=\widetilde{T}_{j} \cap[n, n+1), n \in \mathbb{Z}, j \in \mathbb{N}$. Пусть $F(\cdot, t)=F_{\varepsilon(j)}(\cdot, t)$, если $t \in \widetilde{T}_{j}, j \in \mathbb{N}$, и $F(\cdot, t)=0$, если $t \notin \bigcup_{j} \widetilde{T}_{j}$; $F(\cdot, \cdot) \in B_{1}\left(\Phi_{1}\right)$. Для всех $j \in \mathbb{N}$ имеем

$$
\begin{aligned}
\int_{\widetilde{T}_{j}\left(n_{\varepsilon(j)}\right)}|\mu(F(\cdot, t) ; t)|^{p} d t \geqslant & \int_{T_{\varepsilon(j)}\left(n_{\varepsilon(j)}\right)}\left|\mu\left(F_{\varepsilon(j)}(\cdot, t) ; t\right)\right|^{p} d t \\
& -\sum_{s \geqslant j+1} \int_{T_{\varepsilon(s)}\left(n_{\varepsilon(j)}\right)}\left|\mu\left(F_{\varepsilon(j)}(\cdot, t) ; t\right)\right|^{p} d t \\
\geqslant & \delta-\sum_{s \geqslant j+1} 2^{-1-s+j} \delta=\frac{\delta}{2} .
\end{aligned}
$$

Поэтому $\mu(F(\cdot, \cdot) ; \cdot) \notin M_{p}^{\prime}(\mathbb{R})$ (так как $\left.m\left(T_{\varepsilon(j)}\left(n_{\varepsilon(j)}\right)\right) \leqslant 1 / j\right)$, что противоречит условию леммы. Лемма доказана.

Теорема 6. Пусть $(U, \rho)$ - полное сепарабельное метрическое пространство, $\mathbb{R} \ni t \rightarrow \mu[\cdot ; t] \in \mathscr{M}(U)$ - измеримая мерозначная функция, $p \geqslant 1$. Предположим, ито $|\mu|[U ; \cdot] \in M_{p}(\mathbb{R})$ и для любого $\varepsilon>0$ существует компакт $K_{\varepsilon} \subset U$ такой, что $\left\||\mu|\left[U \backslash K_{\varepsilon} ; \cdot\right]\right\|_{M_{p}(\mathbb{R})}<\varepsilon$. Тогда следующие условия әквивалентны:

1) $\mu[\cdot ; \cdot] \in \widetilde{S}_{p}^{w}(\mathbb{R}, \mathscr{M}(U))$;

2) $\mu[\cdot ; \cdot] \in S_{p}\left(\mathbb{R},\left(\mathscr{M}(U),\|\cdot\|_{w}\right)\right) u|\mu|[U ; \cdot] \in M_{p}^{\prime}(\mathbb{R})$.

При выполнении условий 1) и 2)

$$
\operatorname{Mod} \mu(F(\cdot, \cdot) ; \cdot) \subset \operatorname{Mod} \mu[\cdot ; \cdot]+\sum_{j \in \mathbb{N}} \operatorname{Mod} F\left(\left.\cdot\right|_{K_{\varepsilon_{j}}}, \cdot\right)
$$

для любъх функции $F(\cdot, \cdot) \in \Phi_{\text {ар }}$ и чисел $\varepsilon_{j}>0, j \in \mathbb{N}$, для которых $\varepsilon_{j} \rightarrow 0$ при $j \rightarrow+\infty$. 
ЗАмЕчАнИЕ. Для измеримой функции $t \rightarrow \mu[\cdot ; t] \in \mathscr{M}(U)$ следующие условия эквивалентны:

1) $|\mu|[U ; \cdot] \in M_{p}^{\prime}(\mathbb{R})$ и для любого $\varepsilon>0$ найдется компакт $K_{\varepsilon} \subset U$ такой, что $\left\||\mu|\left[U \backslash K_{\varepsilon} ; \cdot\right]\right\|_{M_{p}(\mathbb{R})}<\varepsilon$

2) $|\mu|[U ; \cdot] \in M_{p}(\mathbb{R})$ и для любого $\varepsilon>0$ существуют измеримое множество $T \subset \mathbb{R}$ и множество $A \in \mathscr{K}_{U}$ такие, что $\sup _{t \in \mathbb{R}} m([t, t+1] \backslash T)<\varepsilon$, $\left\|\chi_{\mathbb{R} \backslash T}(\cdot)|\mu|[U ; \cdot]\right\|_{M_{p}(\mathbb{R})}<\varepsilon$ и $\mu[\cdot ; t] \in A$ для всех $t \in T$.

ДокАЗАТЕЛЬСТво ТЕОРЕмЫ 6 . Так как $\Phi_{\tau} \subset \Phi_{a p}, \tau>0$, то имПЛикация $1) \Rightarrow 2$ ) следует из теоремы 5 и леммы 7. Предположим, что выполнено условие 2$), F(\cdot, \cdot) \in \Phi_{a p}$ и $\varepsilon_{j} \rightarrow 0$ при $j \rightarrow+\infty$. Для любого $j \in \mathbb{N}$ найдутся (см. [7; теорема 2], [19]) измеримые непересекающиеся множества $T_{i}^{(j)} \subset \mathbb{R}$ и функции $F_{i}^{(j)}(\cdot) \in C_{b}(U), i=1, \ldots, n(j)$, такие, что $\chi_{T_{i}^{(j)}}(\cdot) \in S_{1}(\mathbb{R}), \operatorname{Mod} \chi_{T_{i}^{(j)}}(\cdot) \subset$ $\operatorname{Mod} F\left(\left.\cdot\right|_{K_{\varepsilon_{j}}}, \cdot\right), \quad \sup _{t \in \mathbb{R}} m\left([t, t+1] \backslash \bigcup_{i=1}^{n(j)} T_{i}^{(j)}\right)<1 / j, \quad\left\|F_{i}^{(j)}(\cdot)\right\|_{C_{b}(U)} \leqslant$ $\|F(\cdot, \cdot)\|_{\infty}$ и $\left\|F\left(\left.\cdot\right|_{K_{\varepsilon_{j}}}, t\right)-F_{i}^{(j)}\left(\left.\cdot\right|_{K_{\varepsilon_{j}}}\right)\right\|_{C_{b}\left(K_{\varepsilon_{j}}\right)}<1 / j$ для всех $t \in T_{i}^{(j)}$, $i=1, \ldots, n(j) . \quad$ Положим $F_{j}(\cdot, t)=\sum_{i=1}^{n(j)} F_{i}^{(j)}(\cdot) \chi_{T_{i}^{(j)}}(t), t \in \mathbb{R}, j \in \mathbb{N}$. Из теоремы 5 следует, что $\mu\left(F_{i}^{(j)} ; \cdot\right) \in S_{p}(\mathbb{R})$ и $\operatorname{Mod} \mu\left(F_{i}^{(j)} ; \cdot\right) \subset \operatorname{Mod} \mu[\cdot ; \cdot]$. Поэтому $[7 ;$ равенство $(1)$ и лемма 2$] \mu\left(F_{j}(\cdot, \cdot) ; \cdot\right) \in S_{p}(\mathbb{R})$ и

$$
\begin{aligned}
\operatorname{Mod} \mu\left(F_{j}(\cdot, \cdot) ; \cdot\right) & \subset \operatorname{Mod} \mu[\cdot ; \cdot]+\sum_{i=1}^{n(j)} \operatorname{Mod} \chi_{T_{i}^{(j)}}(\cdot) \\
& \subset \operatorname{Mod} \mu[\cdot ; \cdot]+\sum_{j} \operatorname{Mod} F\left(\left.\cdot\right|_{K_{\varepsilon_{j}}}, \cdot\right) .
\end{aligned}
$$

$\mathrm{C}$ другой стороны, из условия $|\mu|[U ; \cdot] \in M_{p}^{\prime}(\mathbb{R})$ получаем, что $\| \mu(F(\cdot, \cdot) ; \cdot)-$ $\mu\left(F_{j}(\cdot, \cdot) ; \cdot\right) \|_{M_{p}(\mathbb{R})} \rightarrow 0$ при $j \rightarrow+\infty$. Следовательно, $\mu(F(\cdot, \cdot) ; \cdot) \in S_{p}(\mathbb{R})$ и вьполнено вложение (9). Теорема доказана.

СлЕДСТВИЕ 1. $\widetilde{S}_{p}^{w}(\mathbb{R}, \mathscr{M}(U))=S_{p}^{\left(\Phi_{\tau}\right)}(\mathbb{R}, \mathscr{M}(U))$ для всех $\tau>0(u p \geqslant 1)$.

ДокАЗАТЕльство. Из вложения $\Phi_{\tau} \subset \Phi_{a p}, \tau>0$, следует $\widetilde{S}_{p}^{w}(\mathbb{R}, \mathscr{M}(U)) \subset$ $S_{p}^{\left(\Phi_{\tau}\right)}(\mathbb{R}, \mathscr{M}(U))$. Пусть $\mu[\cdot ; \cdot] \in S_{p}^{\left(\Phi_{\tau}\right)}(\mathbb{R}, \mathscr{M}(U))$. Так как $\Phi_{0} \subset \Phi_{\tau}$, то $\mu[\cdot ; \cdot] \in$ $S_{p}^{w}(\mathbb{R}, \mathscr{M}(U))$. Из леммы 7 получаем, что $|\mu|[U ; \cdot] \in M_{p}^{\prime}(\mathbb{R})$. Поэтому (а также в силу следствия теоремы 3) выполнено условие 2) теоремы 6 и, следовательно, $\mu[\cdot ; \cdot] \in \widetilde{S}_{p}^{w}(\mathbb{R}, \mathscr{M}(U))$. Следствие доказано.

СлЕДСТВИЕ 2. Если $\mu[\cdot ; \cdot] \in S_{p}^{w}(\mathbb{R}, \mathscr{M}(U)), p \geqslant 1, u \mu[\cdot ; t] \in \mathscr{M}^{+}(U)$ n.в., mo $\mu[\cdot ; \cdot] \in \widetilde{S}_{p}^{w}(\mathbb{R}, \mathscr{M}(U))$.

Приведем пример, показываюший, что существуют мерозначные функции $\mu[\cdot ; \cdot] \in S_{p}(\mathbb{R}, \mathscr{M}(U))$, удовлетворяющие условиям теоремы 6, для которых $|\mu|[U ; \cdot] \notin M_{p}^{\prime}(\mathbb{R})$.

ПримеР 5. Пусть $U=[0,1], \rho(x, y)=|x-y|, x, y \in[0,1], p \geqslant 1$. Определим мерозначные функции $[0,1) \ni t \rightarrow \mu_{l}[\cdot ; t] \in \mathscr{M}(U), l \in \mathbb{N}$ : если $t \in[0,1 / l)$, то 
$\mu_{l}[\mathscr{O} ; t]=\frac{\pi}{2} l^{1 / p} \int_{\mathscr{O}} \sin l \pi x d x, \mathscr{O} \in \mathscr{B}(U) ;$ если $t \in[1 / l, 1)$, то $\mu_{l}[\cdot ; t]=0$. Пусть $\mathscr{A}_{l}=A_{l}+[0,1), l \in \mathbb{N},-$ множества из примера 2. Определим мерозначную функцию $\mu[\cdot ; \cdot]$ : если $t \in \mathscr{A}_{l}, l \in \mathbb{N}$, то положим $\mu[\cdot ; \cdot]=\mu_{l}[\cdot ; t-[t]]$. Так как $\left\|\left|\mu_{l}\right|[U ; \cdot]\right\|_{L_{p}([0,1), \mathbb{R})}=1$ и $\mu_{l}[\cdot ; t]=0$ при $t \in[1 / l, 1)$, то $|\mu|[U ; \cdot] \in M_{p}(\mathbb{R})$ и

$|\mu|[U ; \cdot] \notin M_{p}^{\prime}(\mathbb{R})$. Если $F \in C_{b}(U)$, то $\int_{0}^{1}\left|\mu_{l}(F ; t)\right|^{p} d t \rightarrow 0$ при $l \rightarrow+\infty$, поэтому (см. пример 3) $\mu(F ; \cdot) \in S_{p}(\mathbb{R})$. Следовательно (в силу теоремы 5$), \mu[\cdot ; \cdot] \in$ $S_{p}(\mathbb{R}, \mathscr{M}(U))$.

\section{§4. Доказательство теоремы 2}

Если $\left\{f_{j}\right\} \in Q_{\mathscr{H}}(T), T \neq \varnothing$, то будем использовать (когда это не приводит к недоразумению) краткое обозначение $N_{l}(t)=N_{l}\left[\left\{f_{j}(t)\right\}\right], t \in T, l \in \mathbb{N}$.

Лемма 8. Пусть $\left\{f_{j}\right\} \in Q \mathscr{H}(T), T \neq \varnothing$. Тогда для любого $\varepsilon>0$ и любой последовательности $\left\{t_{l}\right\} \subset T$ можно найти подпоследовательность $\left\{t_{l_{s}}\right\}$ u множество $J \in \mathscr{I}_{1}$ такие, что для всех $s \in \mathbb{N}$

$$
\left\|f\left(J ; t_{l_{s+1}}\right)-f\left(J ; t_{l_{s}}\right)\right\|>\frac{1}{2} N_{l_{s+1}}\left[\left\{f_{j}\left(t_{l_{s+1}}\right)\right\}\right]-\varepsilon .
$$

ДоКАЗАТЕЛЬСТво. Будем последовательно при $s=1,2, \ldots$ находить числа $l_{s} \in \mathbb{N}$ и множества $J_{s} \subset \mathbb{N}$. Положим $l_{1}=1, J_{1}=\{1\}$. Предположим, что числа $l_{k}$ и множества $J_{k}$ уже выбраны для всех $k=1, \ldots, s, s \in \mathbb{N}$. Выберем число $l_{s+1} \in \mathbb{N}$ так, что $l_{s+1}>l_{s}, l_{s+1}>\max \left\{j: j \in \bigcup_{k \leqslant s} J_{k}\right\}$ и $N_{l_{s+1}}\left(t_{l_{s}}\right)<\frac{1}{3} \varepsilon$. Найдется множество $J_{s+1}^{\prime} \in \mathscr{S}_{l_{s+1}}^{\prime}$ такое, что $\left\|f\left(J_{s+1}^{\prime} ; t_{l_{s+1}}\right)\right\|>N_{l_{s+1}}\left(t_{l_{s+1}}\right)-\frac{2}{3} \varepsilon$. Если $\left\|f\left(\bigcup_{k \leqslant s} J_{k} ; t_{l_{s+1}}\right)-f\left(\bigcup_{k \leqslant s} J_{k} ; t_{l_{s}}\right)\right\|>\frac{1}{2} N_{l_{s+1}}\left(t_{l_{s+1}}\right)-\frac{1}{3} \varepsilon$, то положим $J_{s+1}=\varnothing$. В противном случае полагаем $J_{s+1}=J_{s+1}^{\prime}$. В обоих случаях справедливо неравенство

$$
\left\|f\left(\bigcup_{k \leqslant s+1} J_{k} ; t_{l_{s+1}}\right)-f\left(\bigcup_{k \leqslant s} J_{k} ; t_{l_{s}}\right)\right\|>\frac{1}{2} N_{l_{s+1}}\left(t_{l_{s+1}}\right)-\frac{\varepsilon}{3} .
$$

Продолжим неограниченно нахождение чисел $l_{s}$ и множеств $J_{s}\left(J_{s_{1}} \cap J_{s_{2}}=\varnothing\right.$ при $\left.s_{1} \neq s_{2}\right)$. Обозначим $J=\bigcup_{s \in \mathbb{N}} J_{s}$. Для любого $s \in \mathbb{N}$ имеем $\left\|f\left(\bigcup_{k \geqslant s+1} J_{k} ; t_{l_{s}}\right)\right\| \leqslant$ $N_{l_{s+1}}\left(t_{l_{s}}\right)<\frac{1}{3} \varepsilon$. Отсюда и из неравенства (10) для всех $s \in \mathbb{N}$ получаем требуемую оценку

$$
\begin{aligned}
\left\|f\left(J ; t_{l_{s+1}}\right)-f\left(J ; t_{l_{s}}\right)\right\| & \geqslant\left\|f\left(\bigcup_{k \leqslant s+1} J_{k} ; t_{l_{s+1}}\right)-f\left(\bigcup_{k \leqslant s} J_{k} ; t_{l_{s}}\right)\right\| \\
& -\left\|f\left(\bigcup_{k \geqslant s+2} J_{k} ; t_{l_{s+1}}\right)\right\|-\left\|f\left(\bigcup_{k \geqslant s+1} J_{k} ; t_{l_{s}}\right)\right\| \\
& >\frac{1}{2} N_{l_{s+1}}\left(t_{l_{s+1}}\right)-\varepsilon .
\end{aligned}
$$

Лемма доказана. 
Лемма 9. Пусть $\left(V, \rho_{V}\right)$ - компактное метрическое пространство $и\left\{f_{j}\right\} \in$ $Q_{\mathscr{H}}(T)$, әде $T \subset V, T \neq \varnothing$. Предположим, что для всех непустых множеств $J \subset \mathbb{N}$ функции $T \ni t \rightarrow f(J ; t) \in \mathscr{H}$ непрерывны и непрерывно продолжсаются на метрическое пространство $\left(V, \rho_{V}\right)$. Тогда $\lim _{l \rightarrow+\infty} \sup _{t \in T} N_{l}\left[\left\{f_{j}(t)\right\}\right]=0$.

ДокАЗАтЕльство. Допустим противное. Тогда найдутся $\varepsilon>0$ и последовательность $\left\{t_{l}\right\} \subset T$ такие, что $N_{l}\left(t_{l}\right) \geqslant \varepsilon$ для всех $l \in \mathbb{N}$. Переходя, если нужно, к подпоследовательности и учитывая неравенства $N_{l}(t) \geqslant N_{l+1}(t), l \in \mathbb{N}, t \in T$, можно считать, что последовательность $\left\{t_{l}\right\} \subset T \subset V$ сходится при $l \rightarrow+\infty$ к некоторой точке $t_{0} \in V$. В силу леммы 8 найдутся подпоследовательность $\left\{t_{l_{s}}\right\}$ и множество $J \in \mathscr{T}_{1}$ такие, что $\left\|f\left(J ; t_{l_{s+1}}\right)-f\left(J ; t_{l_{s}}\right)\right\|>\frac{1}{2} N_{l_{s+1}}\left(t_{l_{s+1}}\right)-\frac{1}{4} \varepsilon \geqslant \frac{1}{4} \varepsilon$ для всех $s \in \mathbb{N}$. C другой стороны, функция $f(J ; \cdot)$ непрерьвно продолжается на метрическое пространство $\left(V, \rho_{V}\right)$ и $t_{l_{s}} \rightarrow t_{0}$ при $s \rightarrow+\infty$, поэтому $\| f\left(J ; t_{l_{s+1}}\right)-$ $f\left(J ; t_{l_{s}}\right) \| \rightarrow 0$ при $s \rightarrow+\infty$. Полученное противоречие доказывает лемму.

СЛЕДСТВИЕ. Пусть $\left\{f_{j}\right\} \in Q \mathscr{H}([0,+\infty))$. Предположсим, что для всех непустых множсеств $J \subset \mathbb{N}$ функиии $[0,+\infty) \ni t \rightarrow f(J ; t) \in \mathscr{H}$ непрерьвни и существует предел $\lim _{t \rightarrow+\infty} f(J ; t) \in \mathscr{H}$. Тогда $\lim _{l \rightarrow+\infty} \sup _{t \geqslant 0} N_{l}\left[\left\{f_{j}(t)\right\}\right]=0$.

Для доказательства следствия достаточно заметить, что функции $[0, \pi / 2) \ni$ $\varphi \rightarrow f_{j}(\operatorname{tg} \varphi), j \in \mathbb{N}$, удовлетворяют условиям леммы 9 (при этом $\left.V=[0, \pi / 2]\right)$.

Непрерывная функция $f: \mathbb{R} \rightarrow \mathscr{H}$ имеет среднее значение $M(f)$, если существует предел

$$
\lim _{\eta \rightarrow+\infty} \frac{1}{2 \eta} \int_{-\eta}^{\eta} f(t) d t=M(f) \in \mathscr{H} .
$$

Лемма 10. Пусть $\left\{f_{j}\right\} \in Q \mathscr{H}(\mathbb{R})$ и для любого множсества $J \in \mathscr{S}_{1}$ функиии $f(J ; \cdot)$ непрерьвны и имеют среднее значение $M(f(J ; \cdot)) \in \mathscr{H}$. Определим непрерывные функции

$$
g_{j}(\eta)=\frac{1}{2 \eta} \int_{-\eta}^{\eta} f_{j}(t) d t, \quad \eta>0, \quad g_{j}(0)=f_{j}(0), \quad j \in \mathbb{N} .
$$

Тогда $\left\{g_{j}\right\} \in Q \mathscr{H}([0,+\infty)) u \sup _{\eta \geqslant 0} N_{l}\left[\left\{g_{j}(\eta)\right\}\right] \rightarrow 0$ nрu $l \rightarrow+\infty$.

ДоказАтельство. Для любого множества $J \in \mathscr{S}_{1}$ обозначим

$$
g(J ; \eta)=\frac{1}{2 \eta} \int_{-\eta}^{\eta} f(J ; t) d t, \quad \eta>0, \quad g(J ; 0)=f(J ; 0) .
$$

Функции $g(J ; \cdot)$ непрерывны. Из леммы 9 следует, что $\sum_{j \in J, j \leqslant l} f_{j}(t) \rightarrow f(J ; t)$ при $l \rightarrow+\infty$ равномерно на каждом отрезке $[-\eta, \eta], \eta \geqslant 0$. Поэтому

$$
\sum_{j \in J, j \leqslant l} g_{j}(\eta) \rightarrow \sum_{j \in J} g_{j}(\eta)=g(J ; \eta)
$$

при $l \rightarrow+\infty$ для всех $\eta \geqslant 0$. Отсюда получаем $\left\{g_{j}\right\} \in Q \mathscr{H}([0,+\infty))$. Равенство $\lim _{l \rightarrow+\infty} \sup _{\eta \geqslant 0} N_{l}\left[\left\{g_{j}(\eta)\right\}\right]=0$ вытекает из следствия леммы 9, так как каждая функция $\eta \rightarrow g(J ; \eta), J \in \mathscr{S}_{1}$, имеет предел $M(f(J ; \cdot)) \in \mathscr{H}$ при $\eta \rightarrow+\infty$. Лемма доказана. 
ТЕОРема 7. Пусть $(\mathscr{H},\|\cdot\|)$ - (вещественное или комплексное) банахово пространство, $\left\{f_{j}\right\} \in Q \mathscr{H}(\mathbb{R})$. Предположим, что $f(J ; \cdot) \in C A P(\mathbb{R}, \mathscr{H})$ для всех непустых мнохеств $J \subset \mathbb{N}$. Тогда $\operatorname{Mod} f(J ; \cdot) \subset \sum_{j} \operatorname{Mod} f_{j}(\cdot)$ для всех $J \subset \mathbb{N}, J \neq \varnothing$.

ДокАЗАТЕЛЬство. Удобно считать пространство $\mathscr{H}$ комплексньм. Поэтому в случае вешественного пространства $\mathscr{H}$ будем рассматривать его комплексификацию (условия теоремы от этого не изменятся). Пусть $\lambda \in \mathbb{R}$ и $\lambda \notin \sum_{j} \operatorname{Mod} f_{j}$. Обозначим $f_{j}^{\lambda}(t)=e^{-i \lambda t} f_{j}(t), t \in \mathbb{R},\left\{f_{j}^{\lambda}\right\} \in Q_{\mathscr{H}}(\mathbb{R})$. Для любого множества $J \in \mathscr{S}_{1}$ функция $t \rightarrow e^{-i \lambda t} f(J ; t)=f^{\lambda}(J ; t)=\sum_{j \in J} f_{j}^{\lambda}(t), t \in \mathbb{R}$, принадлежит пространству $C A P(\mathbb{R}, \mathscr{H})$ и, следовательно, имеет среднее значение. Обозначим

$$
\begin{gathered}
g^{\lambda}(J ; \eta)=\frac{1}{2 \eta} \int_{-\eta}^{\eta} f^{\lambda}(J ; t) d t, \quad \eta>0, \quad g^{\lambda}(J ; 0)=f(J ; 0), \quad J \in \mathscr{S}_{1} \\
g_{j}^{\lambda}(\cdot)=g^{\lambda}(\{j\} ; \cdot), \quad j \in \mathbb{N} .
\end{gathered}
$$

Пусть $J \in \mathscr{S}_{1} \backslash \mathscr{S}_{l+1}, l \in \mathbb{N}$. Справедливо неравенство

$$
\sup _{\eta \geqslant 0}\left\|g^{\lambda}(J ; \eta)-g^{\lambda}(J \cap\{1, \ldots, l\} ; \eta)\right\| \leqslant \sup _{\eta \geqslant 0} N_{l+1}\left[\left\{g_{j}^{\lambda}(\eta)\right\}\right]
$$

Так как модуль $\operatorname{Mod} f$ любой функции $f \in C A P(\mathbb{R}, \mathscr{H})$ совпадает с модулем ее показателей Фурье [11], [12], то из выбора числа $\lambda$ следует, что $g^{\lambda}(J \cap\{1, \ldots, l\} ; \eta)$ $\rightarrow 0$ при $\eta \rightarrow+\infty$. Поэтому

$$
\left\|M\left(f^{\lambda}(J ; \cdot)\right)\right\| \leqslant \sup _{\eta \geqslant 0} N_{l+1}\left[\left\{g_{j}^{\lambda}(\eta)\right\}\right]
$$

В силу леммы 10 отсюда (при $l \rightarrow+\infty)$ для любого непустого множества $J \subset \mathbb{N}$ получаем $M\left(f^{\lambda}(J ; \cdot)\right)=0$. Поэтому любое число $\lambda \in \mathbb{R} \backslash \sum_{j} \operatorname{Mod} f_{j}$ не является показателем Фурье функции $f(J ; \cdot) \in C A P(\mathbb{R}, \mathscr{H})$ и, следовательно, $\operatorname{Mod} f(J ; \cdot) \subset$ $\sum_{j} \operatorname{Mod} f_{j}$. Теорема 7 доказана.

Перейдем непосредственно к доказательству теоремы 2. Выберем любой счетньй плотный в $\mathbb{R}$ модуль $\Lambda=\left\{\lambda_{\nu}, \nu \in \mathbb{N}\right\}$, для которого $\sum_{j} \operatorname{Mod} f_{j} \subset \Lambda$. Определим на $\mathbb{R}$ метрику

$$
\rho_{\Lambda}\left(t_{1}, t_{2}\right)=\sum_{\nu} 2^{-\nu}\left|e^{i \lambda_{\nu} t_{1}}-e^{i \lambda_{\nu} t_{2}}\right|, \quad t_{1}, t_{2} \in \mathbb{R} .
$$

Пополнение $\left(B_{\Lambda}, \rho_{\Lambda}\right)$ метрического пространства $\left(\mathbb{R}, \rho_{\Lambda}\right)$ компактно (относительная компактификация Бора [20; гл. 1]). Функции $f \in C A P(\mathbb{R}, \mathscr{H})$, для которых $\operatorname{Mod} f \subset \Lambda$, равномерно непрерывны на $\left(\mathbb{R}, \rho_{\Lambda}\right)$ и, следовательно, продолжаются до непрерывных функций на метрическом пространстве $\left(B_{\Lambda}, \rho_{\Lambda}\right)$. Поэтому из теоремы 7 следует, что для всех непустых множеств $J \subset \mathbb{N}$ функции $\left(\mathbb{R}, \rho_{\Lambda}\right) \ni t \rightarrow f(J ; t) \in \mathscr{H}$ непрерывны и имеют непрерьвное продолжение на метрический компакт $B_{\Lambda}$. Осталось воспользоваться леммой 9. Теорема доказана. 


\section{§5. Доказательство теоремы 4}

Лемма 11. Пусть $\left\{f_{j}\right\} \in \widetilde{Q} \mathscr{H}(T)$, где $T \subset \mathbb{R}-$ измеримое множество, для которого $0<m(T)<+\infty$. Тогда для любих $\varepsilon>0$ и $\delta>0$ существует такое $l \in \mathbb{N}$, что для любого множсества $J \in \mathscr{S}_{l}^{\prime}$ справедливо неравенство $m(\{t \in T:\|f(J ; t)\| \geqslant \varepsilon\})<\delta$.

ДоказАтельство. Допустим противное. Тогда найдутся числа $\varepsilon, \delta>0$ такие, что для каждого $l \in \mathbb{N}$ сушествует множество $J \in \mathscr{S}_{l}^{\prime}$, для которого $m(\{t \in T:$ $\|f(J ; t)\| \geqslant \varepsilon\}) \geqslant \delta$. Отсюда следует существование множеств $J_{s} \in \mathscr{S}_{1}^{\prime}, s \in \mathbb{N}$, таких, что $\max \left\{j: j \in J_{s}\right\}<\min \left\{j: j \in J_{s+1}\right\}$ и $m\left(\left\{t \in T:\left\|f\left(J_{s} ; t\right)\right\| \geqslant \varepsilon\right\}\right) \geqslant \delta$ для всех $s \in \mathbb{N}$. Множество $\widetilde{T}=\bigcap_{l \in \mathbb{N}} \bigcup_{s \geqslant l}\left\{t \in T:\left\|f\left(J_{s} ; t\right)\right\| \geqslant \varepsilon\right\}$ измеримо и $m(\widetilde{T}) \geqslant \delta$. Обозначим $J^{\prime}=\bigcup_{s \in \mathbb{N}} J_{s}$. Если $t \in \widetilde{T}$, то $\left\|f\left(J_{s} ; t\right)\right\| \geqslant \varepsilon$ для бесконечного числа индексов $s$, поэтому ряд $\sum_{j \in J^{\prime}} f_{j}(t)$ для всех $t \in \widetilde{T}$ не сходится. Последнее (так как $m(T)>0$ ) противоречит предположению $\left\{f_{j}\right\} \in \widetilde{Q}_{\mathscr{H}}(T)$. Лемма доказана.

TЕОрема 8. Пусть $\left\{f_{j}\right\} \in \widetilde{Q}_{\mathscr{H}}(T)$, әде $T \subset \mathbb{R}-$ измеримое множество, для которого $0<m(T)<+\infty, p \geqslant 1$ и для любого непустого множества $J \subset \mathbb{N}$ функиия $T \ni t \rightarrow f(J ; t)=\sum_{j \in J} f_{j}(t)$ принадлежсит $L_{p}(T, \mathscr{H})$. Тогда

$$
\lim _{l \rightarrow+\infty} \sup _{J \in \mathscr{S}_{l}^{\prime}} \int_{T}\|f(J ; t)\|^{p} d t=0
$$

ДоказАтельство. Допустим противное. Тогда найдется такое $\varepsilon>0$, что для любого $l \in \mathbb{N}$ сушествует множество $J \in \mathscr{S}_{l}^{\prime}$, для которого $\int_{T}\|f(J ; t)\|^{p} d t \geqslant \varepsilon^{p}$. Положим $l_{1}=1, T_{1}=T$. Выберем множество $J_{1} \in \mathscr{S}_{1}^{\prime}$, для которого

$$
\int_{T}\left\|f\left(J_{1} ; t\right)\right\|^{p} d t \geqslant \varepsilon^{p} .
$$

Сушествует число $\delta_{1}>0$ такое, что для всех измеримых множеств $T^{\prime} \subset T$, для которых $m\left(T^{\prime}\right) \leqslant \delta_{1}$, выполняется неравенство

$$
\int_{T^{\prime}}\left\|f\left(J_{1} ; t\right)\right\|^{p} d t \leqslant \frac{1}{4} \varepsilon^{p} .
$$

Будем далее последовательно при $s=2,3, \ldots$ определять числа $\delta_{s}, \delta_{s}^{\prime}>0, l_{s} \in \mathbb{N}$, множества $J_{s} \in \mathscr{S}_{l_{s}}^{\prime}$ (для которых $l_{s}<l_{s+1}$ и $\max \left\{j: j \in J_{s}\right\}<\min \left\{j: j \in J_{s+1}\right\}$ при всех $s \in \mathbb{N}$ ) и измеримые множества $T_{s} \subset T$. Предположим, что они уже определены для некоторого $s \in \mathbb{N}$ (число $\delta_{1}^{\prime}$ не определяется). Найдем число $\delta_{s+1}^{\prime}>0$ такое, что $\delta_{s+1}^{\prime}<2^{-k} \delta_{s+1-k}$ для всех $k=1, \ldots, s$ и

$$
\int_{T^{\prime \prime}}\left\|f\left(\bigcup_{k \leqslant s} J_{k} ; t\right)\right\|^{p} d t \leqslant 2^{-2 p} \varepsilon^{p}
$$


для всех измеримых множеств $T^{\prime \prime} \subset T$, для которых $m\left(T^{\prime \prime}\right) \leqslant \delta_{s+1}^{\prime}$. Выберем число $l_{s+1} \in \mathbb{N}$ так, чтобы выполнялись следующие два условия:

1) $l_{s+1}>\max \left\{j: j \in J_{s}\right\}$;

2) для любого множества $J \in \mathscr{S}_{l_{s+1}^{\prime}}^{\prime}$ справедливо неравенство

$$
m\left(\left\{t \in T:\|f(J ; t)\| \geqslant 2^{-s-2}(m(T))^{-1 / p} \varepsilon\right\}\right) \leqslant \delta_{s+1}^{\prime}
$$

(последнего можно добиться в силу леммы 11).

После этого множество $J_{s+1} \in \mathscr{S}_{l_{s+1}^{\prime}}^{\prime}$ выберем так, чтобы выполнялось неравенство

$$
\int_{T}\left\|f\left(J_{s+1} ; t\right)\right\|^{p} d t \geqslant \varepsilon^{p} .
$$

Найдем такое число $\delta_{s+1}>0$, что

$$
\int_{T^{\prime}}\left\|f\left(J_{s+1} ; t\right)\right\|^{p} d t \leqslant \frac{1}{4} \varepsilon^{p}
$$

для всех измеримых множеств $T^{\prime} \subset T$, для которых $m\left(T^{\prime}\right) \leqslant \delta_{s+1}$. Положим $T_{s+1}=\left\{t \in T:\left\|f\left(J_{s+1} ; t\right)\right\| \geqslant 2^{-s-2}(m(T))^{-1 / p} \varepsilon\right\}$. Тогда $m\left(T_{s+1}\right) \leqslant \delta_{s+1}^{\prime}$ и, следовательно,

$$
\int_{T_{s+1}}\left\|f\left(\bigcup_{k \leqslant s} J_{k} ; t\right)\right\|^{p} d t \leqslant 2^{-2 p} \varepsilon^{p} .
$$

Продолжим неограниченно нахождение чисел $\delta_{s}, \delta_{s}^{\prime}, l_{s}$ и множеств $J_{s} \in \mathscr{S}_{l_{s}}^{\prime}, T_{s} \subset T$. Положим $T_{s}^{\prime}=T_{s} \backslash \bigcup_{k>s} T_{k}, s \in \mathbb{N}$. Множества $T_{s}^{\prime}$ при разных $s$ не пересекаются. Так как $m\left(T_{k}\right) \leqslant \delta_{k}^{\prime}$ для всех $k \geqslant 2$, то $m\left(\bigcup_{k>s} T_{k}\right) \leqslant \sum_{k \geqslant s+1} 2^{s-k} \delta_{s}=\delta_{s}$. Поэтому из (12)-(15) получаем

$$
\int_{T_{s}^{\prime}}\left\|f\left(J_{s} ; t\right)\right\|^{p} d t \geqslant \varepsilon^{p}-\frac{1}{4} \varepsilon^{p} \geqslant\left(\frac{3 \varepsilon}{4}\right)^{p}, \quad s \in \mathbb{N} .
$$

При п.в. $t \in T_{s}^{\prime}, s \in \mathbb{N}$, имеем $\left\|f\left(G_{k} ; t\right)\right\|<2^{-k-1}(m(T))^{-1 / p} \varepsilon$ для всех $k \geqslant s+1$. Обозначим $J=\bigcup_{s \in \mathbb{N}} J_{s}$. Из (15) и (16) вытекают неравенства

$$
\begin{aligned}
\left(\int_{T_{s}^{\prime}}\|f(J ; t)\|^{p} d t\right)^{1 / p} \geqslant & \left(\int_{T_{s}^{\prime}}\left\|f\left(J_{s} ; t\right)\right\|^{p} d t\right)^{1 / p}-\left(\int_{T_{s}^{\prime}}\left\|f\left(\bigcup_{k<s} J_{k} ; t\right)\right\|^{p} d t\right)^{1 / p} \\
& -\sum_{k \geqslant s+1}\left(\int_{T_{s}^{\prime}}\left\|f\left(J_{k} ; t\right)\right\|^{p} d t\right)^{1 / p} \\
\geqslant & \frac{3 \varepsilon}{4}-\frac{\varepsilon}{4}-\sum_{k \geqslant s+1} 2^{-k-1} \varepsilon \geqslant \frac{\varepsilon}{4}, \quad s \in \mathbb{N}
\end{aligned}
$$

(второй интеграл в средней части неравенств отсутствует при $s=1$ ). Так как множества $T_{s}^{\prime} \subset T, s \in \mathbb{N}$, не пересекаются, то отсюда получаем

$$
\int_{T}\|f(J ; t)\|^{p} d t=+\infty
$$

что противоречит условиям теоремы. Теорема 8 доказана. 
ЗАмечАниЕ. В условиях теоремы 8 для любого множества $J \in \mathscr{S}_{l}, l \in \mathbb{N}$, имеем $\sum_{j \in J, j \leqslant k} f_{j}(t) \rightarrow f(J ; t)$ п.в. при $k \rightarrow+\infty$. Поэтому из леммы Фату следует, что для всех $l \in \mathbb{N}$

$$
\sup _{J \in \mathscr{S}_{l}^{\prime}} \int_{T}\|f(J ; t)\|^{p} d t=\sup _{J \in \mathscr{S}_{l}} \int_{T}\|f(J ; t)\|^{p} d t .
$$

Перейдем непосредственно к доказательству теоремы 4. Рассмотрим преобразование Бохнера, ставящее в соответствие функциям $\mathbb{R} \ni t \rightarrow f(t) \in \mathscr{H}$ из пространства $S_{p}(\mathbb{R}, \mathscr{H})$ функции $\mathbb{R} \ni t \rightarrow f(\cdot+t) \in L_{p}([0,1], \mathscr{H})$ из пространства $C A P\left(\mathbb{R}, L_{p}([0,1], \mathscr{H})\right)$. Из теоремы 8 (и замечания после нее) следует, что для любого непустого множества $J \subset \mathbb{N}$

$$
\left\|\sum_{j \in J, j \leqslant l} f_{j}(\cdot+t)-\sum_{j \in J} f_{j}(\cdot+t)\right\|_{L_{p}([0,1], \mathscr{H})} \rightarrow 0
$$

при $l \rightarrow+\infty$, поэтому $\left\{f_{j}(\cdot+t)\right\} \in Q_{L_{p}([0,1], \mathscr{H})}$ и $f(J ; \cdot+t)=\sum_{j \in J} f_{j}(\cdot+t)$ (ряд сходится в пространстве $\left.L_{p}([0,1], \mathscr{H})\right)$ для всех $t \in \mathbb{R}$. Так как для любого непустого множества $J \subset \mathbb{N}$ функция $t \rightarrow f(J ; \cdot+t)$ принадлежит пространству $C A P\left(\mathbb{R}, L_{p}([0,1], \mathscr{H})\right)$, то доказываемое утверждение следует из теоремы 2 . Теорема доказана.

\section{Список литературы}

1. Ширяев А. Н. Вероятность. М.: Наука, 1989.

2. Красовский Н. Н. Управление динамической системой. М.: Наука, 1985.

3. Ченцов А. Г. Приложение теории меры к задачам управления. Свердловск, 1985.

4. Варга Дж. Оптимальное управление дифференциальными и функциональньми уравнениями. М.: Наука, 1977.

5. Иванов А.Г. Оптимальное управление почти периодическими движениями // ПММ. 1992. T. 56. № 5. C. 133-142.

6. Иванов А. Г. Об оптимальном управлении почти периодическими движениями при наличии ограничений на средние типа равенств и неравенств. I, II, III // Дифференц. уравнения. 1997. Т. 33. № 2. С. 167-176; №3. С. 316-323; №4. С. 478-485.

7. Данилов Л.И. Мерозначные почти периодические функции и почти периодические сечения многозначных отображений // Матем. сб. 1997. Т. 188. № 10. С. 3-24.

8. Пинни Э. Обыкновенные дифференциально-разностные уравнения. М.: ИЛ, 1961.

9. Мышкис $A$. Д. Линейные дифференциальные уравнения с запаздывающим аргументом. М.: Наука, 1972.

10. Бутковский А. Г. Методы управления системами с распределенньми параметрами. М.: Наука, 1975.

11. Левитан Б. М. Почти-периодические функции. М.: ГИТТЛ, 1953.

12. Левитан Б. М., Жиков В.В. Почти-периодические функции и дифференциальные уравнения. М.: Изд-во МГУ, 1978.

13. Вахания Н.Н., Тариеладзе В.И., Чобанян С.А. Вероятностные распределения в банаховых пространствах. М.: Наука, 1985.

14. Данилов Л. И. Мерозначные почти периодические функции // Матем. заметки. 1997. Т. 61. № 1. С. 57-68.

15. Данилов Л. И. О равномерной аппроксимации почти периодических по Степанову функций // Изв. вузов. Сер. матем. 1998. № 5. С. 10-18.

16. Красносельский М. А., Бурд В. Ш., Колесов Ю. С. Нелинейные почти периодические колебания. М.: Наука, 1970. 
17. Данилов Л.И. Об операторах суперпозиции, сохраняющих почти периодичность // Деп. в ВИНИТИ. 26.05.98. № 1589-В98.

18. Данилов Л. И., Иванов А. Г. К теореме о поточечном максимуме в почти периодическом случае // Изв. вузов. Сер. матем. 1994. №6. С. 50-59.

19. Данилов Л. И. Почти периодические сечения многозначных отображений // Изв. отдела математики и информатики УдГУ. № 1. Ижевск, 1993. С. 16-78.

20. Панков A. А. Ограниченные и почти периодические решения нелинейных дифференциально-операторных уравнений. Киев: Наукова думка, 1985.

Физико-технический институт

Поступила в редакцию

Удмуртского научного центра УрО РАН, Ижевск

10.01 .1999 и 13.04 .2000 